Acta Botanica Mexicana 67: 1-41 (2004)

\title{
CATÁLOGO DE EJEMPLARES TIPO DEL HERBARIO IEB (MÉXICO)*
}

\author{
Gilberto Ocampo Acosta y Ana Lilia Medellín Villamil \\ Instituto de Ecología, A.C. Centro Regional del Bajío Apdo. postal 386, \\ C.P. 61600, Pátzcuaro, Mich. e-mail: ocampo@inecolbajio.edu.mx
}

\section{RESUMEN}

El presente trabajo provee un catálogo que comprende los ejemplares tipo de plantas vasculares que alberga el herbario del Instituto de Ecología, A.C., en Pátzcuaro, Mich. (IEB). La colección incluye 340 especímenes designados como tipos primarios ( 85 holotipos, 247 isotipos, 2 neotipos y 6 isoneotipos), los cuales representan la referencia nomenclatural de 286 taxa.

Palabras clave: herbario IEB, México, tipos nomenclaturales.

\begin{abstract}
In this paper a catalog of type specimens of vascular plants held in the herbarium of the Instituto de Ecología, A.C., located in Pátzcuaro, Michoacán, Mexico (IEB), is provided. The collection includes 340 specimens designed as primary types ( 85 holotypes, 247 isotypes, 2 neotypes and 6 isoneotypes), which represent the nomenclatural reference for 286 taxa.
\end{abstract}

Key words: herbarium IEB, Mexico, nomenclatural types.

\section{INTRODUCCIÓN}

Un ejemplar tipo representa, según el Código Internacional de Nomenclatura Botánica vigente (Greuter et al., 2000), el elemento al cual el nombre de un taxon está permanentemente ligado, por lo que su revisión es de gran importancia para

\footnotetext{
*Trabajo realizado con apoyo económico del Instituto de Ecología, A. C. (cuenta 902-07), del Consejo Nacional de Ciencia y Tecnología y de la Comisión Nacional para el Conocimiento y Uso de la Biodiversidad.
} 
la aplicación correcta del epíteto científico. Por desgracia, existe escasa literatura en la que se ponga de manifiesto cuales son los ejemplares tipo que alojan los herbarios mexicanos, aunque cabe destacar que en fechas relativamente recientes algunas colecciones tales como IBUG (Martínez, 1993) y ENCB (Arreguín et al., 1996; Arreguín et al., 2003) han hecho público el catálogo de sus acervos de tipos, así como MEXU que da a conocer su inventario de tipos nomenclaturales de manera paulatina en una serie de publicaciones parciales (e.g. Hernández y Lira, 1991; Lira et al., 1992; Lira et al., 1996; Olvera, 1999; Montero et al., 2001). A pesar de que no se ha divulgado la lista de tipos de la gran mayoría de los herbarios mexicanos, las colecciones consideradas por Llorente et al. como las más importantes del país (p. 45, cuadro 9, 1999) cuentan con datos muy aceptables acerca de tales ejemplares que se encuentran bajo su resguardo, condición que seguramente prevalece en la generalidad de las instituciones nacionales. En el Cuadro 1 se presenta una sinopsis numérica del estado actual de las colecciones de ejemplares tipo de los once herbarios más grandes de México (se consideran únicamente los tipos primarios, excluyendo así los paratipos, topotipos y fototipos).

De acuerdo con los datos anteriores, la colección de tipos del herbario IEB del Instituto de Ecología, A.C., establecido en Pátzcuaro, Mich., es una de las más grandes del país, superada en número únicamente por la de AMO, la de ENCB y la de MEXU. La colección se compone hasta el momento de un total de 340 ejemplares designados como tipos primarios de plantas vasculares mexicanas, los cuales representan la referencia nomenclatural de 286 taxa (Cuadro 2), la mayoría de ellos ubicados en el grupo de Angiospermas (276). A continuación se muestra información acerca de las familias mejor representadas (Cuadro 3), los estados mexicanos con mayor número de tipos (Cuadro 4), datos acerca del material tipo colectado para la zona de estudio del Bajío y de regiones adyacentes (Cuadro 5), así como los colectores con mayor número de ejemplares en el acervo (Cuadro 6).

En seguida, se presenta el catálogo de los tipos primarios depositados en el herbario IEB, dispuestos en orden alfabético por familia, género y especie dentro de cada grupo (cabe destacar que el arreglo por familias que aquí se presenta refleja la manera en que se encuentra ordenada la colección general del herbario). Para cada taxon se muestran los siguientes datos: nombre científico y autor (es); referencia de la publicación original; estado, municipio y localidad de colecta; fecha de colecta; colector y número de colecta, así como la clase de tipo nomenclatural a la que corresponde el ejemplar. Si el espécimen fue colectado en la zona de estudio del Bajío y de regiones adyacentes, el símbolo $\$$ se antepone al nombre genérico. Adicionalmente, se muestra información acerca de sinonimias, en el caso de que éstas existan, además de incluir en algunos casos particulares un breve comentario. 
Ocampo y Medellín: Catálogo de Ejemplares Tipo del Herbario IEB

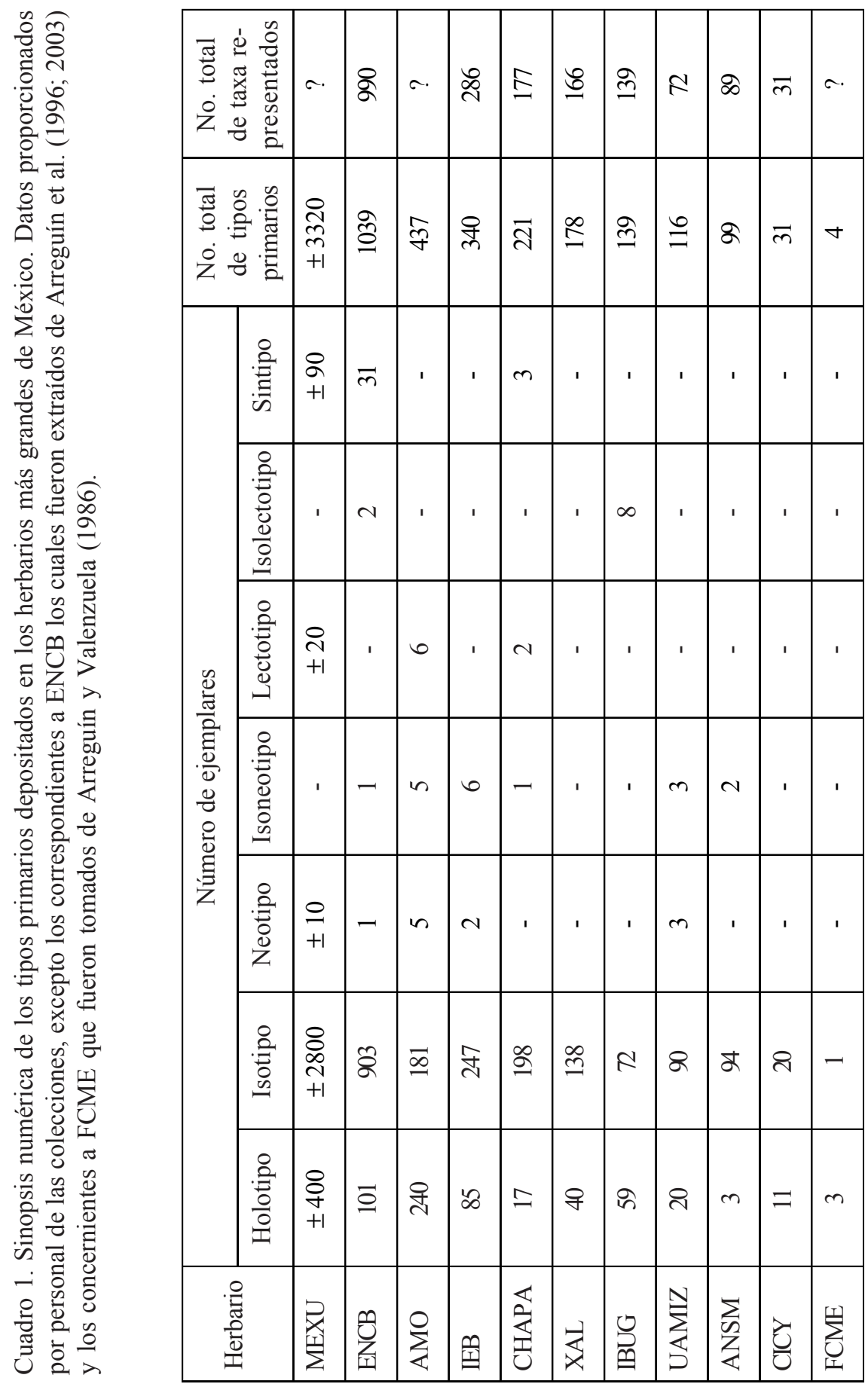


Cuadro 2. Número de taxa representados por categoría taxonómica en la colección de tipos del herbario IEB.

\begin{tabular}{|l|c|}
\hline \multicolumn{1}{|c|}{ Categoría taxonómica } & $\begin{array}{c}\text { Número de taxa representados } \\
\text { por ejemplares tipo }\end{array}$ \\
\hline Especie & 264 \\
Subespecie & 5 \\
Variedad & 16 \\
Forma & 1 \\
\hline Total & 286 \\
\hline
\end{tabular}

Cuadro 3. Familias mejor representadas en el acervo de tipos nomenclaturales de IEB.

\begin{tabular}{|l|c|}
\hline \multicolumn{1}{|c|}{ Familia } & Número de taxa \\
\hline Compositae & 68 \\
Euphorbiaceae & 14 \\
Burseraceae & 13 \\
Lentibulariaceae & 13 \\
Crassulaceae & 11 \\
Iridaceae & 11 \\
Cyperaceae & 9 \\
Leguminosae & 9 \\
Liliaceae & 9 \\
\hline
\end{tabular}


Cuadro 4. Estados mejor representados en la colección de tipos de IEB.

\begin{tabular}{|l|c|}
\hline \multicolumn{1}{|c|}{ Estado } & Número de taxa \\
\hline Oaxaca & 41 \\
Querétaro & 39 \\
Nuevo León & 35 \\
Jalisco & 28 \\
Durango & 24 \\
Michoacán & 24 \\
Guanajuato & 19 \\
Guerrero & 15 \\
Hidalgo & 12 \\
\hline
\end{tabular}

Cuadro 5. Número de taxa representados por categoría taxonómica en la colección de tipos de IEB que corresponden a ejemplares colectados en la zona de estudio del Bajío y de regiones adyacentes. Entre paréntesis, se destaca el número de taxa que han sido descritos como resultado del trabajo de exploración intensiva y sistemática por parte del personal ligado al IEB.

\begin{tabular}{|l|c|c|c|c|}
\hline & Familia & Género & Especie & $\begin{array}{c}\text { Categorías } \\
\text { infraespecíficas }\end{array}$ \\
\hline Guanajuato & 13 & 18 & $18(16)$ & $1(1)$ \\
Michoacán & 13 & 15 & $15(12)$ & $1(1)$ \\
Querétaro & 26 & $31(1)$ & $37(30)$ & $2(2)$ \\
\hline Total & 52 & $64(1)$ & $70(58)$ & $4(4)$ \\
\hline
\end{tabular}


Acta Botanica Mexicana 67: 1-41 (2004)

Cuadro 6. Colectores con mayor número de ejemplares en la colección de tipos del herbario IEB.

\begin{tabular}{|l|c|}
\hline \multicolumn{1}{|c|}{ Colector } & Número de ejemplares \\
\hline J. Rzedowski & 39 \\
Hinton et al. & 36 \\
S. Zamudio & 20 \\
R. McVaugh & 19 \\
S. González & 13 \\
\hline
\end{tabular}

\section{PTERIDOPHYTA}

Polypodiaceae

Asplenium stolonipes Mickel \& Beitel, Mem. New York Bot. Gard. 46: 72.1988. OAXACA: distrito de Ixtlán: trail N of San Pedro Nolasco to the Llano Verde, at the high point above the Llano Verde (Las Cruces), 8.X.1970, J. T. Mickel y S. W. Leonard 5376. Isotipo

Elaphoglossum alan-smithii Mickel, Brittonia 32: 334. 1980. OAXACA: distrito de Ixtlán: trail from San Pedro Nolasco to the Llano Verde, 4.X.1969, J. T. Mickel 3739. Isotipo

Grammitis oidiophora Mickel \& Beitel, Mem. New York Bot. Gard. 46: 202. 1988. OAXACA: distrito de Ixtlán: trail from San Pedro Nolasco N to the Llano Verde, 5.X.1969, J. T. Mickel y R. L. Hellwig 3822. Isotipo

Hypolepis blepharochlaena Mickel \& Beitel, Mem. New York Bot. Gard. 46: 219. 1988. OAXACA: distrito de Teotitlán: 26-29 km NE of Teotitlán del Camino, vicinity of pass at Puerto Soledad, 16.X.1969, J. T. Mickel y R. L. Hellwig 4151. Isotipo 
Ocampo y Medellín: Catálogo de Ejemplares Tipo del Herbario IEB

Pellaea ribae Mendoza \& Windham, Acta Bot. Mex. 57: 16. 2001. SAN LUIS POTOSÍ: municipio de San Nicolás Tolentino: $3 \mathrm{~km}$ después del Puerto de Santa Gertrudis, rumbo a Buenavista, 15.XI.1997, A. Mendoza, J. Ceja, A. Espejo y A. R. López-Ferrari 250. Isotipo

\$ Polypodium microgrammoides Mickel \& A. R. Smith, Brittonia 52: 236: 2000. GUANAJUATO: municipio de Ocampo: \pm 4 km al S de Santa Bárbara, 7.X.1992, E. Carranza et al. 4320. Holotipo

Selaginellaceae

\$ Selaginella arsiclada Valdespino, Amer. Fern. J. 84: 99. 1994. QUERÉTARO: municipio de Landa: orilla del río Moctezuma, Tangojó, 4.III.1988, H. Díaz B. 4627. Isotipo

Selaginella basipilosa Valdespino, Brittonia 44: 314. 1992. OAXACA: distrito de Tlacolula: E of Oaxaca on route 190 near km 36, 26.IX.1970, J. T. Mickel y S. W. Leonard 4965. Isotipo

Selaginella corrugis Mickel \& Beitel, Mem. New York Bot. Gard. 46: 336.1988. OAXACA: distrito de Villa Alta: valley of the Yelagago River, ca. $20 \mathrm{mi} \mathrm{NE}$ of Villa Alta, woods $\mathrm{N}$ of Hallberg ranch, 27.VII.1962, J. T. Mickel 1043. Isotipo

\section{GYMNOSPERMAE}

Pinaceae

Pinus luzmariae Pérez de la Rosa, Bol. Inst. Bot. (Guadalajara) 5: 127. 1998. DURANGO: municipio de Mezquitic: $10 \mathrm{~km}$ al S de Tenzompa, camino a Los Amoles, 28.VII.1994, J. A. Pérez de la Rosa 1839. Isotipo

\section{ANGIOSPERMAE}

Acanthaceae

$\$$ Carlowrightia venturae Daniel, Novon 13: 37. 2003. GUANAJUATO: municipio de Victoria: Cerro de La Luz, camino a Xichú, 26.VII.1991, E. Ventura y E. López 9336. Holotipo e Isotipo 
† Justicia zamudioi T. F. Daniel, Novon 13: 40. 2003. QUERÉTARO: municipio de Landa: $6 \mathrm{~km}$ al E de La Vuelta, 21.IX.1988, S. Zamudio 6814. Holotipo e Isotipo

Poikilacanthus novogalicianus Daniel, Bull. Torrey Bot. Club 118: 455. 1991. COLIMA: 8 miles WNW of Santiago, road to Cihuatlán, Jalisco, 25-26.VII.1957, R. McVaugh, J. T. Mickel y M. R. McVaugh 15759. Isotipo

+ Tetramerium carranzae T. F. Daniel, Novon 13: 43. 2003. GUANAJUATO: municipio de Xichú: $3 \mathrm{~km}$ NE de El Guamúchil, 22.XII.1997, E. Carranza y $P$. Cervantes 5470. Holotipo

Amaryllidaceae

† Agave tenuifolia Zamudio \& E. Sánchez, Acta Bot. Mex. 32: 48. 1995. QUERÉTARO: municipio de Jalpan: 6-7 km de La Parada, camino a El Cañón, 29.VII.1989, E. Carranza 1905. Holotipo

Furcraea guerrerensis Matuda ( $=F$. pubescens Tod.), An. Inst Biol. Univ. Méx. 36: 144. 1965. GUERRERO: municipio de Chilpancingo: Rincón Viejo, 8.VIII.1964, H. Kruse 865a. Isotipo

Hypoxis lucens McVaugh, Fl. Novo-Galiciana 15: 225. 1989. JALISCO: Sierra del Tigre, 3 miles S of Mazamitla, 17.IX.1952, McVaugh 13000 (en el ejemplar se cita: R. McVaugh y J. Sooby, Jr. 13000). Isotipo

Polianthes geminiflora (Lex.) Rose var. clivicola McVaugh, Fl. Novo-Galiciana 15: 250. 1989. JALISCO: slopes facing the Pacific 10 miles S of Autlán, 5.VIII.1949, R. L. Wilbur y C. R. Wilbur 2133. Isotipo

† Polianthes multicolor E. Solano \& Dávila, Novon 13: 119. 2003. GUANAJUATO: municipio de San Luis de la Paz: sobre el camino viejo de terracería a Xichú, en la base del cerro Garbanzo (en el ejemplar se cita: sobre el camino de terracería a Xichú, faldas del cerro Garbanzo), 16.VIII.1996, E. Solano y C. Correa 871. Isotipo

Anacardiaceae

\$ Cotinus carranzae Rzedowski \& Calderón, Acta Bot. Mex. 47: 24. 1999. QUERÉTARO: municipio de Landa: río Moctezuma, al S de Matzacintla, 29.X.1998, E. Pérez C., E. Carranza y R. Spellenberg 3903. Holotipo e Isotipo 
Ocampo y Medellín: Catálogo de Ejemplares Tipo del Herbario IEB

Annonaceae

Cymbopetalum hintonii Lundell ssp. septentrionale N. A. Murray, Syst. Bot. Monogr. 40: 31. 1993. JALISCO: between El Tuito and Boca de Tomatlán on Hwy 200, 6.0 mi S of Boca de Tomatlán, 28.VI.1983 (en el ejemplar se cita: 27.VI.1983), N. A. Murray y D. M. Johnson 1456. Isotipo

Araceae

Anthurium kruseanum Matuda (= A. nizandense Matuda), An. Inst Biol. Univ. Méx. 36: 107. 1965. GUERRERO: municipio de Juan Escudero: La Junta, cerca de Tierra Colorada, en la unión de los ríos Omitlán y Papagayo, 18.VII.1965, H. Kruse 881. Isotipo

Aristolochiaceae

Aristolochia colimensis Santana-Michel, Acta Bot. Mex. 58: 64. 2002. COLIMA: municipio de Colima: La Salada, $17 \mathrm{~km}$ al S de Colima, 19.XI.1998, F. J. Santana Michel y S. Lemus J. 9068. Isotipo

Berberidaceae

\$ Berberis albicans Zamudio \& Marroquín, Southwest. Nat. 32: 483. 1987. QUERÉTARO: municipio de Cadereyta: $500 \mathrm{~m}$ al W de Vizarrón, 29.VI.1979, $S$. Zamudio 3666. Isotipo

Bromeliaceae

Pitcairnia flavescentia Matuda, An. Inst. Biol. Univ. Mex. 36: 110. 1965. GUERRERO: municipio de Chilpancingo: Rincón de la Vía, 3.IX.1961, H. Kruse 464. Isotipo

\$ Pitcairnia vallisoletana Lex., Nov. Veg. Descr. 1: 19. 1824. (Neotipo designado por A. Espejo, A. R. López-Ferrari y M. Flores en Acta Bot. Mex. 23: 55. 1993). MICHOACÁN: municipio de Morelia: $3.6 \mathrm{~km}$ después de Jesús del Monte, sobre el camino a San Miguel del Monte, 8.IV.1992, M. Flores Cruz, A. R. López-Ferrari y A. Espejo 740. Isoneotipo 
Acta Botanica Mexicana 67: 1-41 (2004)

Burseraceae

Amiris linanoé La Llave (= Bursera linaloe (La Llave) Rzedowski, Calderón \& Medina), Reg. Trim. 1: 356. 1832. (Neotipo designado por J. Rzedowski, R. Medina y G. Calderón en Acta Bot. Mex. 66: 103. 2004). OAXACA: distrito de Teotitlán: camino a Huautla de Jiménez (en el ejemplar se cita municipio de Teotitlán del Camino), 12.VII.1978, F. Ventura 15356. Neotipo e Isoneotipo

Bursera altijuga Rzedowski, Calderón \& Medina, Acta Bot. Mex. 66: 38.2004. OAXACA: distrito de Coixtlahuaca: Puerto Mixteco, $4 \mathrm{~km}$ al N de Tepelmeme, 19.IV.1966 (en el ejemplar se cita: 19.IV.1969), R. Cruz 2362. Holotipo e Isotipo

Bursera esparzae Rzedowski, Calderón \& Medina, Acta Bot. Mex. 66: 77. 2004. OAXACA: distrito de Etla: municipio de Telixtlahuaca: El Parián, cerca del cementerio, 13.VI.2002, J. Rzedowski 53888. Holotipo e Isotipo

Bursera heliae Rzedowski \& Calderón, Acta Bot. Mex. 59: 83. 2002. OAXACA: distrito de Tlacolula: Portillo de San Dionisio, 13 km al SSE de Matatlán, 10.VIII.1976, J. Rzedowski 34385. Holotipo e Isotipo

Bursera isthmica Rzedowski \& Calderón, Acta Bot. Mex. 59: 86. 2002. OAXACA: distrito de Tehuantepec: municipio de Salina Cruz: Cerro Cuachi al W de Salina Cruz, 30.VIII.1985, C. Martínez 175. Holotipo

Bursera laurihuertae Rzedowski \& Calderón, Acta Bot. Mex. 52: 75. 2000. OAXACA: municipio de Totolapan: $3 \mathrm{~km}$ al NE de San Juan Guegoyache, 9.VIII.1976, J. Rzedowski 34383. Holotipo e Isotipo

Bursera macvaughiana Cuevas \& Rzedowski, Acta Bot. Mex. 46: 78. 1999. JALISCO: municipio de Tuxcacuesco: en el Cerro del Palacio, 4-5 km al SW de Tuxcacuesco, 9.VII.1997, R. Cuevas y R. Delgado 5619. Holotipo e Isotipo

Bursera martae Jiménez R. \& Cruz-Durán, Acta Bot. Mex. 55: 7. 2001. GUERRERO: municipio de General Heliodoro Castillo: $1 \mathrm{~km}$ al NE de Tecomazúchil, 9.VII.2000, R. Cruz Durán, J. Castrejón y M. García 4799. Isotipo

Bursera medranoana Rzedowski \& Ortiz, Acta Bot. Mex. 1: 17. 1988. HIDALGO: municipio de Cardonal: fondo de la Barranca de Tolantongo, 30.V.1984, J. Rzedowski 38401a. Isotipo 
Bursera palaciosii Rzedowski \& Calderón, Acta Bot. Mex. 50: 47. 2000. JALISCO: municipio de La Huerta: Estación de Biología Chamela, cerca de Chamela, 26.VII.1982, J. Rzedowski 37882. Holotipo e Isotipo

Bursera pontiveteris Rzedowski, Calderón \& Medina, Acta Bot. Mex. 66: 116. 2004. OAXACA: distrito de Cuicatlán: municipio de Santiago Nacaltepec: arriba del río Las Ventas, de regreso a Puente Viejo, 22.X.2002, R. Medina et al. 1279. Holotipo e Isotipo

Bursera ribana Rzedowski \& Calderón, Acta Bot. Mex. 50: 51. 2000. JALISCO: municipio de Tomatlán: $6 \mathrm{~km}$ al S de Tomatlán, sobre la carretera a La Cumbre, 27.VII.1982, J. Rzedowski 37886. Holotipo e Isotipo

Bursera vazquezyanesii Rzedowski \& Calderón, Acta Bot. Mex. 50: 55. 2000. JALISCO: municipio de Cabo Corrientes: $14 \mathrm{~km}$ al W de El Tuito, sobre el camino a Llano Grande de Ipala, 27.VII.1982, J. Rzedowski 37888. Holotipo e Isotipo

Buxaceae

† Buxus moctezumae Köhler, Fernández \& Zamudio, Feddes Repert. 104: 295. 1993. QUERÉTARO: municipio de Landa: $15 \mathrm{~km}$ al SE de Agua Zarca, 25.V.1990, $R$. Fernández y S. Zamudio 4617. Isotipo

Cactaceae

Ariocarpus bravoanus H. Hernández \& E. Anderson, Bradleya 10: 1. 1992. SAN LUIS POTOSÍ: municipio de Guadalcázar: torre de microondas Núñez, a $79 \mathrm{~km}$ al N de San Luis Potosí por la carretera San Luis Potosí - Matehuala, 19.VIII.1991, H. Hernández, 1977. Isotipo

Aztekium hintonii Glass \& W. A. Fitz Maurice, Cact. Suc. Mex. 37: 13. 1992. NUEVO LEÓN: municipio de Galeana: localidad no especificada para evitar saqueo, 26.IX.1991, Hinton et al. s. n. Holotipo e Isotipo

Geohintonia mexicana Glass \& W. A. Fitz Maurice, Cact. Suc. Mex. 37: 17. 1992. NUEVO LEÓN: municipio de Galeana: localidad no especificada para evitar saqueo, 26.IX.1991, Hinton et al. s. n. Isotipo

Mammillaria luethyi G. S. Hinton, Phytologia 80: 58. 1996. COAHUILA: municipio de Acuña: localidad no especificada para evitar saqueo, 19.V.1996, Hinton et al. 25771. Isotipo 
* Mammillaria scheinvariana R. Ortega-Varela \& Glass, Guía Ident. Cact. Amenaz. México 1: Ma/sche. 1998. QUERÉTARO: municipio de Cadereyta: Mesa de León, presa de Zimapán, 15.III.1997, rescate Zimapán s. n. Holotipo

+ Turbinicarpus alonsoi Glass \& Arias, Kakteen Sukk. 47: 26. 1996. GUANAJUATO: $7.5 \mathrm{~km}$ de Xichú, en una curva cerca de la mina Aurora y del ejido Río Abajo, 20.XII.1994, A. García y C. Glass 024. Holotipo

Turbinicarpus booleanus G. S. Hinton, Phytologia 80: 62. 1996. NUEVO LEÓN: municipio de Galeana: Y Griega (en el ejemplar se cita: near Y), 1.III.1992, Hinton et al. 21805. Isotipo

Campanulaceae

Lobelia flexicaulis Rzedowski \& Calderón, Acta Bot. Mex. 55: 35. 2001. GUERRERO: municipio de Taxco: $7 \mathrm{~km}$ al SSE de Tetipac, sobre el camino a Taxco, 18.XI.2000, J. Rzedowski 53779. Holotipo e Isotipo

Lobelia guerrerensis Eakes \& Lammers, Novon 9: 381. 1999. GUERRERO: municipio de Atoyac de Álvarez: a 6 km al SW de Puerto del Gallo, 29.III.1983, J. C. Soto y E. Martínez 5136. Isotipo

‡ Lobelia orientalis Rzedowski \& Calderón, Acta Bot. Mex. 40: 62. 1997. QUERÉTARO: municipio de Landa: Llano Chiquito, 1-2.IV.1997, S. Zamudio y E. Carranza 10200. Holotipo e Isotipo

Lobelia porphyrea Rzedowski \& Calderón, Acta Bot. Mex. 55: 33. 2001. HIDALGO: municipio de Zimapán: cañón del arroyo Tolimán, $\pm 3 \mathrm{~km}$ al S de San Cristóbal, 27.VIII.1999, S. Zamudio 11099. Holotipo

Capparidaceae

Cleome chapalaensis H. H. Iltis, Bol. Inst. Bot. (Guadalajara) 5: 428. 1998. MICHOACÁN: on road to La Barca, $1 / 3 \mathrm{~km} \mathrm{~N}$ of the R.R. Station at Zamora, 29.VII.1960, H. H. Iltis, R. Koeppen y F. Iltis 490. Isotipo

Caprifoliaceae

$\$$ Abelia grandifolia Villarreal, Brittonia 52. 174. 2000. QUERÉTARO: municipio de Jalpan: 9-10 km al S de San Juan de los Durán, Cerro Grande, 13.VI.1991, B. Servín 1101. Holotipo 
Abelia mexicana Villarreal, Brittonia 52: 172. 2000. OAXACA: distrito de Juxtlahuaca: municipio de San Sebastián Tecomaxtlahuaca: $10 \mathrm{~km}$ de San Sebastián Tecomaxtlahuaca, carretera a San Martín Duraznos, 19.VII.1996, J. I. Calzada 21100. Isotipo

Caryophyllaceae

Arenaria gypsostrata B. L. Turner, Phytologia 75: 481. 1993 (nombre substituto para A. hintoniorum B. L. Turner, Phytologia 75: 400. 1993, nom. ileg., no Arenaria hintoniorum B. L. Turner, 1992). NUEVO LEÓN: municipio de Aramberri: along road from La Escondida to Aramberri, 23.X.1993, Hinton et al. 23706. Isotipo

Cistaceae

+ Helianthemum pugae Calderón, Acta Bot. Mex. 16: 89. 1991. GUANAJUATO: municipio de Ocampo: $10 \mathrm{~km}$ al S de Ibarra, sobre la carretera a León, 3.VIII.1991, J. Rzedowski 50792. Isotipo

Commelinaceae

Callisia guerrerensis Matuda (publicado como C. guerrerense), An. Inst Biol. Univ. Méx. 37: 78. 1966. GUERRERO: municipio de Juan Escudero: Tierra Colorada, en sombra parcial de acantilado orientado al W de la barranca formada por el río Omitlán, 1.XI.1965, H. Kruse 905. Isotipo

Commelina nivea López-Ferrari, Espejo \& Ceja, Acta Bot. Mex. 41: 10. 1997. DURANGO: municipio de Pueblo Nuevo: Las Adjuntas, km 120 de la carretera Durango - Mazatlán, $20 \mathrm{~km}$ después de El Salto, rumbo a La Ciudad (en el ejemplar se cita: 20 km después de El Salto, rumbo a Mazatlán), 7.VIII.1996, A. Espejo, A. R. López-Ferrari, J. Ceja y A. Mendoza R. 5458. Isotipo

Commelina rzedowskii López-Ferrari, Espejo \& Ceja, Acta Bot. Mex. 41: 12. 1997. ESTADO DE MÉXICO: municipio de Tejupilco: aproximadamente 2-3 km al NW de Tenería, rumbo a Río Grande, 1.VIII.1996, A. R. López-Ferrari y A. Espejo 2339. Isotipo

Commelina socorrogonzaleziae Espejo \& López-Ferrari, Sida 15: 441. 1993. DURANGO: municipio de El Salto: 2 km después de Coyotes, sobre la desviación a San Miguel de Cruces, 28.VII.1991, M. Flores Cruz, A. Espejo y A. R. LópezFerrari 502. Isotipo 
Acta Botanica Mexicana 67: 1-41 (2004)

Compositae

Acourtia acevedoi M. González-Elizondo (= A. macrocephala Sch. Bip. ex Seemann), Phytologia 61: 117. 1986. DURANGO: municipio de Vicente Guerrero: Rincón de Las Mulas, a 3 km al SW de San Isidro, 16.II.1985, S. Acevedo 163. Isotipo

Acourtia bravohollisiana Rzedowski, Bol. Soc. Bot. Méx. 45: 98. 1983. GUERRERO: camino a la torre de microondas Tuxpan, $\pm 10 \mathrm{~km}$ al NE de Iguala, 23.XII.1980, J. Rzedowski 37162. Isotipo

Acourtia butandae L. Cabrera R., Brittonia 53: 423. 2001. CHIHUAHUA: $18 \mathrm{~km}$ S of Villa Matamoros, hwy. 45, 24.VIII.1988, L. Cabrera R. y G. Dieringer 650. Isotipo

+ Acourtia carranzae L. Cabrera R., Brittonia 53: 416. 2001. QUERÉTARO: municipio de Arroyo Seco: aprox. $1 \mathrm{~km}$ al S de Arroyo Seco, 21.II.1989, E. Carranza 1488. Holotipo e Isotipo

Acourtia ciprianoi Panero \& Villaseñor, Brittonia 51: 87. 1999. OAXACA: distrito de Tehuantepec: municipio de Santiago Astata: Santiago Astata, carretera Pochutla - Salina Cruz, 18.X.1988, C. Martínez 1978. Isotipo

Acourtia discolor Rzedowski, Bol. Soc. Bot. Méx. 45: 100. 1983. OAXACA: 30 km al NNW de Telixtlahuaca, sobre la carretera a Cuicatlán, 6.I.1981, J. Rzedowski 37181. Isotipo

† Acourtia elizabethiae Rzedowski \& Calderón, Acta Bot. Mex. 12: 16. 1990. QUERÉTARO: municipio de Cadereyta: alrededores de Maconí, 10.XI.1988, J. Rzedowski 47625. Isotipo

Acourtia fragrans Rzedowski, Bol. Soc. Bot. Méx. 45: 102. 1983. PUEBLA: Salinas Grandes, cerca de Zapotitlán de las Salinas, 6.I.1981, J. Rzedowski 37174. Isotipo

Acourtia hidalgoana B. L. Turner, Phytologia 74: 394. 1993. HIDALGO: 7 km al NE de Mezquititlán, sobre la carretera a Zacualtipán, 17.XI.1974, J. Rzedowski 32510. Isotipo 
Acourtia hintoniorum B. L. Turner, Phytologia 75: 404. 1993. NUEVO LEÓN: municipio de Aramberri: between La Escondida and Josecito, 13.XI.1993, Hinton et al. 23934. Isotipo

Acourtia huajuapana B. L. Turner var. actinomorpha Rzedowski, Bol. Soc. Bot. Méx. 45: 104. 1983. GUERRERO: $4 \mathrm{~km}$ al S de Zumpango del Río, sobre la carretera a Chilpancingo, 23.XII.1980, J. Rzedowski 37160. Isotipo

Acourtia intermedia L. Cabrera R., Brittonia 53: 418. 2001. DURANGO: $166 \mathrm{mi}$ NW of Durango, along hwy. 145, 24.VIII.1988, L. Cabrera R. y G. Dieringer 653. Holotipo

\$ Acourtia joaquinensis L. Cabrera R., Brittonia 53: 423. 2001. QUERÉTARO: municipio de San Joaquín: ca. $1 \mathrm{~km}$ al S de Santo Tomás, 24.X.1991, V. M. Huerta 1324. Holotipo

† Acourtia moctezumae Rzedowski \& Calderón, Acta Bot. Mex. 12: 14. 1990. QUERÉTARO: municipio de Landa: $4 \mathrm{~km}$ al SE de Matzacintla, 18.XII.1987, L. M. Chávez 217. Isotipo

\$ Acourtia queretarana B. L. Turner (= A. carpholepis (Sch. Bip. ex A. Gray) Reveal \& R. M. King), Phytologia 74: 402. 1993. QUERÉTARO: cerca de Huazmazontla, $13 \mathrm{~km}$ al NE de Pinal de Amoles, sobre la carretera a Jalpan, 15.I.1989, J. Rzedowski 48108. Isotipo

\$ Acourtia venturae L. Cabrera R., Brittonia 53: 420. 2001. GUANAJUATO: municipio de Victoria: Puerto del Aire, por la Sierra de Victoria, 12.XI.1991, E. Ventura y E. López 9797. Holotipo e Isotipo

Ageratina acevedoana B. L. Turner, Phytologia 75: 402. 1993. DURANGO: $10 \mathrm{~km}$ de Canelas, por el camino a Santiago Papasquiaro, 10.III.1987, S. Acevedo y D. Bayona 190. Isotipo

Ageratina gonzaleziorum B. L. Turner (publicado como A. gonzalezorum), Phytologia 58: 498. 1985. DURANGO: municipio de El Mezquital: 19 km de Los Charcos, por el camino a Santa María Ocotán, 4.X.1983, S. González y M. González 2558. Isotipo

Ageratina gypsophila B. L. Turner, Phytologia 57: 130. 1985. NUEVO LEÓN: municipio de Galeana: $10 \mathrm{~km}$ NE Pocitos (en el ejemplar se cita: $10 \mathrm{~km}$ E of Pocitos), 26.VIII.1984, Hinton et al. 18779. Isotipo 
Ageratina ilicifolia B. L. Turner, Phytologia 71: 311. 1991. NUEVO LEÓN: municipio de Rayones: $19 \mathrm{~km}$ from Galeana along the road to Rayones, 20.X.1990, Hinton et al. 20837. Isotipo

Ageratina josepaneroi B. L. Turner, Phytologia 81: 344. 1996. GUERRERO: municipio de Chilpancingo: $\mathrm{km} 8$ de la carretera Chilpancingo - Chichihualco, 8.X.1995, J. L. Panero y C. Clevinger 6183. Isotipo

Ageratina juxtlahuacensis Panero \& Villaseñor, Lundellia 1: 72. 1998. OAXACA: distrito de Juxtlahuaca: km 15 de la carretera Tecomaxtlahuaca - Coicoyán de Las Flores, 17.X.1994, J. L. Panero, E. Manrique e I. Calzada 5102. Isotipo

Ageratina pendula Panero \& Villaseñor, Phytologia 48: 498. 1996. OAXACA: distrito de Santiago Juxtlahuaca: municipio de Santiago Juxtlahuaca: $16 \mathrm{~km}$ al S de Santiago Juxtlahuaca sobre la carretera Santiago Juxtlahuaca - Putla y aproximadamente $1 \mathrm{~km}$ al $\mathrm{S}$ de este punto a lo largo de un riachuelo, 31.V.1995, J. L. Panero, I. Calzada y C. Clevinger 5686. Isotipo

Arnicastrum guerrerense Villaseñor, Syst. Bot. 11: 277. 1986. GUERRERO: municipio de Tlacotepec: Cerro Teotepec, $12 \mathrm{~km}$ al NE de Puerto del Gallo, 19.X.1983, J. C. Soto, E. Martínez y G. Silva 5863. Isotipo

Axiniphyllum durangense B. L. Turner, Madroño 34: 165. 1987. DURANGO: municipio de El Mezquital: alrededores de Plátano Tatemado (a $12 \mathrm{~km}$ de La Guajolota), 12.IX.1985, I. Solís 294. Isotipo

+ Baccharis zamoranensis Rzedowski, Brittonia 24: 400. 1972. QUERÉTARO: municipio de Colón: parte más alta del Cerro Zamorano, 13.XI.1971, J. Rzedowski y R. McVaugh 425. Isotipo

Carminatia alvarezii Rzedowski \& Calderón, Anales Esc. Nac. Ci. Biol. 31: 9. 1987. OAXACA: $5 \mathrm{~km}$ al E de Teotitlán del Camino, sobre la carretera a Huautla, 25.X.1980, J. Rzedowski 37075. Isotipo

Chaptalia hidalgoensis L. Cabrera \& G. L. Nesom, Sida 20: 1363. 2003. HIDALGO: municipio de Zimapán: Verdosas, 20.XI.1991, V. M. Huerta 1352. Holotipo

\$ Chrysactinia luzmariae Rzedowski \& Calderón, Bol. Inst. Bot. (Guadalajara) 5: 61. 1998. GUANAJUATO: municipio de Xichú: 5 km al E de Las Adjuntas, sobre el camino a Huamúchil, 12.VII.1997, J. Rzedowski 53447. Holotipo 
Ocampo y Medellín: Catálogo de Ejemplares Tipo del Herbario IEB

\$ Cirsium bicentenariale Rzedowski, Acta Bot. Mex. 29. 101. 1994. QUERÉTARO: municipio de Pinal de Amoles: $7 \mathrm{~km}$ al NE de Pinal de Amoles, sobre el camino a Jalpan, 18.III.1987, J. Rzedowski 42872. Holotipo

\$ Cirsium zamoranense Rzedowski, Acta Bot. Mex. 29: 104. 1994. QUERÉTARO: municipio de Colón: parte alta del Cerro Zamorano, 1.IX.1987, J. Rzedowski 44443. Holotipo

Coreopsis bolanosana Panero \& Villaseñor, Brittonia 51: 89. 1999. JALISCO: municipio de Bolaños: $10 \mathrm{~km}$ al SW de Banderitas o en el crucero Tuxpan de Bolaños - Los Amoles, 27.IX.1996, J. Calónico-Soto y G. Flores F. 3006. Isotipo

Coreopsis mutica DC. var. miahuatlana B. L. Turner, Phytologia 80: 293. 1996. OAXACA: municipio de Miahuatlán: Santo Domingo, IRF Río Magdalena (en el ejemplar se cita: Santo Domingo Ozolotepec), 4.VIII.1996, Hinton et al. 26724. Isotipo

Desmanthodium hintoniorum B. L. Turner, Phytologia 79: 317. 1995. OAXACA: municipio de Miahuatlán: La Sirena, 23.X.1995, Hinton et al. 26409. Isotipo

Erigeron chiangii Nesom var. lamprocaulis Nesom, Phytologia 73: 119. 1992. COAHUILA: municipio de Múzquiz: ca. 130 road km NW of Muzquiz along Coahuila Hwy 2-A (Muzquiz-Boquillas), then into Sierra La Encantada, bottom of deep, N-S trending canyon on SW side of La Encantada basin, Sierra Buenavista of the Sierra Encantada, on SE side of road going up into mining area of the sierra (en el ejemplar se cita: ca. 130 road km NW of Muzquiz on Coa Hwy 2A (Mzq.Boquillas), then SW of $2 \mathrm{~A}$ on tunnel road to La Encantada mining area, SW side of La Encantada basin, Sa. Buenavista of Sa. La Encantada), 3.VI.1992, G. Nesom y M. Mayfield 7408. Isotipo

Eupatorium guiengolense Torres Colín \& J. L. Villaseñor, Acta Bot. Mex. 23:47. 1993. OAXACA: distrito de Tehuantepec: municipio de Santo Domingo Tehuantepec: Cerro Guiengola, $11 \mathrm{~km}$ al NW de Tehuantepec, carretera a Oaxaca, 9.XII.1991, A. Campos V. 4234. Isotipo

Flaveria kochiana B. L. Turner, Phytologia 78: 400. 1995. OAXACA: municipio de San Agustín Atenango: $14 \mathrm{~km}$ al SW de San Marcos Arteaga, carr. Huajuapan - Juxtlahuaca, 23.XI.1989, S. D. Koch y P. A. Fryxell 89221. Isotipo 
Laennecia spellenbergii Nesom, Phytologia 73: 267. 1992. DURANGO: municipio de Súchil: ca. 47 air km SSW of Vicente Guerrero on road to Las Margaritas, on the Reserva de la Biosfera "La Michilía", on general S slope of Cerro Blanco, 23.VII.1990, R. Spellenberg y S. González E. 10285. Isotipo

Montanoa laskowskii McVaugh, Contr. Univ. Mich. Herb. 9: 423. 1972. JALISCO: 16 miles SW of La Huerta, along road between Autlán and Barra de Navidad (Mex. highway 80), 26.IX.1966, W. R. Anderson y C. W. Laskowski 3777. Isotipo

+ Perezia fruticosa La Llave \& Lex. (= Acourtia fruticosa (La Llave \& Lex.) B. L. Turner), Nov. Veg. Descr. 1: 24. 1824. (Neotipo designado por B. L. Turner en Phytologia 74: 390. 1993). MICHOACÁN: hills, Jesús del Monte near Morelia, 29.XI.1907, C. G. Pringle 10410. Isoneotipo

Perymenium hintoniorum B. L. Turner, Phytologia 71: 315. 1991. NUEVO LEÓN: municipio de Rayones: ca. $24 \mathrm{~km}$ from Galeana along the road to Rayones, 17.X.1990, Hinton et al. 20788. Isotipo

Perymenium hintoniorum B. L. Turner var. gypsophilum B. L. Turner, Phytologia 79: 343. 1995. NUEVO LEÓN: municipio de Aramberri: along road from Aramberri to El Salitre, 26.X.1993, Hinton et al. 23749. Isotipo

Psacalium hintoniorum B. L. Turner, Phytologia 80: 92. 1996. OAXACA: municipio de Miahuatlán: Neverías, 6.I.1996, Hinton et al. 26557. Isotipo

Psacalium perezii B. L. Turner, Phytologia 67: 419. 1989. JALISCO: municipio de Tecalitlán: $50.7 \mathrm{~km}$ al SSE de Ciudad Guzmán, carretera a Llanitos y brecha a Plan de Lego y $3 \mathrm{~km}$ al $\mathrm{S}$ de P. de Lego, 4.VIII.1988, M. Fuentes O. 486. Isotipo

Psathyrotopsis hintoniorum B. L. Turner, Phytologia 75: 143. 1993. COAHUILA: municipio de Parras: S of Parras, 1.III.1993, Hinton et al. 22692. Isotipo

Sabazia liebmannii Klatt. var. ovatifolia Longpre, Publ. Mus. Michigan State Univ., Biol. Ser. 4: 362. 1970. OAXACA: along hwy. 190 between km 422 \& 423, ca. 47 miles W of Oaxaca, 23.VIII.1961, E. K. Longpre 199. Isotipo

Senecio cyclophyllus Greenman (= Senecio coahuilensis Greenman), Publ. Field Columb. Mus., Bot. 2: 276. 1907. NUEVO LEÓN: Sierra Madre above Monterrey, 31.III.1906, C. G. Pringle 10230. Isotipo 
Ocampo y Medellín: Catálogo de Ejemplares Tipo del Herbario IEB

Senecio floresiorum B. L. Turner, Phytologia 74: 367. 1993. JALISCO: municipio de Totatiche: Rancho Acaspulco, $8 \mathrm{~km}$ al SW de Temastián, 28.VI.1991, A. Flores M. y J. Ma. Flores M. 2688. Isotipo

Senecio gonzaleziae B. L. Turner (publicado como S. gonzalezae), Phytologia 57: 377. 1985. DURANGO: municipio de El Mezquital: $3 \mathrm{~km}$ al S de Santa María de Ocotán, 17.X.1984, M. González y S. Acevedo 1558. Isotipo

Senecio mezquitalanus B. L. Turner, Phytologia 71: 56.1996 (nombre substituto para S. gesnerifolius B. L. Turner, Phytologia 62: 75. 1987, nom. ileg., no $S$. gesnerifolius Cuatrecasas, 1950). DURANGO: municipio de El Mezquital: $26.5 \mathrm{~km}$ de La Guajolota por el camino a Platanitos, 15.III.1985, M. González et al. 1677. Isotipo

Senecio rzedowskii García-Pérez (= S. jacalensis Greenman), Phytologia 57: 275. 1985. ESTADO DE MÉXICO: municipio de Tlalmanalco: Llano Atlihuiyán, 10 km al E de San Rafael, 18.VII.1982, J. Rzedowski 37876. Isotipo

Senecio tapianus B. L. Turner, Phytologia 67: 387. 1989. VERACRUZ: municipio de Chiconquiaco: cima del Cerro del Borrego, entre Planta del Pie y Buenavista, 21.I.1988, L. Tapia y J. Hernández 517. Isotipo

Solidago wrightii A. Gray var. orientalis Nesom (= S. orientalis (Nesom) Nesom), Phytologia 67: 147. 1989. COAHUILA: municipio de Arteaga: Sierra La Marta, 6.IX.1981, Hinton et al. 18333. Isotipo

\$ Tagetes moorei H. Robinson var. breviligulata Villarreal, Brittonia 54: 165. 2002. QUERÉTARO: municipio de San Joaquín: cañada de La Culebra, $\pm 3 \mathrm{~km}$ al NE de La Tinaja, 20.X.1994, S. Zamudio y E. Pérez C. 9391. Holotipo

Tehuana calzadae Panero \& Villaseñor, Syst. Bot. 21: 555. 1996. OAXACA: distrito de Tehuantepec: municipio de San Pedro Huamelula: $7.6 \mathrm{~km}$ al N de la carretera Pochutla - Salina Cruz, sobre la carretera a Cocos a través de San Pedro Huamelula, 17.IX.1995, J. L. Panero, I. Calzada y T. Boland 5970. Isotipo

Tridax durangensis A. García-Arévalo, Acta Bot. Mex. 12: 9. 1990. DURANGO: municipio de El Mezquital: aprox. 4 km de Candelarita Chico, 21.VIII.1988, I. Solís 958. Isotipo 
Verbesina aramberrana B. L. Turner, Phytologia 75: 134. 1993. NUEVO LEÓN: municipio de Aramberri: San Francisco (en el ejemplar se cita: near San Francisco), 26.VIII.1992, Hinton et al. 22359. Isotipo

Verbesina durangensis B. L. Turner, Phytologia 60: 254. 1986. DURANGO: municipio de El Mezquital: alrededores de La Guajolota, 16.VIII.1985, I. Solís 229. Isotipo

Verbesina nayaritensis B. L. Turner, Phytologia 63: 10. 1987. NAYARIT: municipio de Huajicori: 2 km del Rancho de Los Sauces, 8.XI.1985, I. Solís 560. Isotipo

Verbesina pellucida Villaseñor \& Panero, Contr. Univ. Mich. Herb. 19: 93. 1993. OAXACA: km 10 of the road to Santa María Chimalapa, ca. $10 \mathrm{~km}$ E of the intersection of this road and the road Palomares - Juchitán, 17.II.1992, J. L. Panero y A. Campos 2746. Isotipo

Vernonia diazlunana B. L. Turner, Phytologia 69: 169. 1990. JALISCO: municipio de Tecalitlán: Río San Pedro, 20.XI.1986, C. L. Díaz Luna 18179. Isotipo

Vernonia pugana Jones \& Stutts, Brittonia 33: 544. 1981. JALISCO: foothills of Sierra de Manantlán and narrow rocky stream-valley along road to summit-pass, 16$22 \mathrm{~km} \mathrm{~S}$ of El Chante, 3-6.II.1975, R. McVaugh, G. D. McPherson y R. B. McVaugh 26172. Isotipo

† Vernonia solorzanoana Rzedowski \& Calderón, Acta Bot. Mex. 32: 7. 1995. MICHOACÁN: municipio de Morelia: $2 \mathrm{~km}$ al $\mathrm{S}$ de San José de las Torres, 29.III.1987, J. Rzedowski 42903. Holotipo e Isotipo

Viguiera grammatoglossa DC. var. huajuapana Panero \& Villaseñor, Brittonia 48: 571. 1996. OAXACA: municipio de Huajuapan de León: $\mathrm{km} 32.2$ de la carretera Huajuapan de León - Juxtlahuaca, 29.IX.1995, J. L. Panero, I. Calzada y C. Clevinger 6141. Isotipo

Viguiera inegii S. González, M. González-Elizondo \& J. Rzedowski, Acta Bot. Mex. 53: 39. 2000. DURANGO: municipio de Rodeo: ca. $1 \mathrm{~km}$ al NE de Las Ánimas, sobre la carretera a Nazas, 5.VIII.1997, S. González, M. González y S. Acevedo 5907. Holotipo e Isotipo 
Viguiera oligantha S. González, M. González-Elizondo \& J. Rzedowski, Acta Bot. Mex. 53: 37. 2000. DURANGO: municipio de Rodeo: ca. 1 km al NE de Las Ánimas, sobre la carretera a Nazas, 5.VIII.1997, S. González, M. González y S. Acevedo 5909. Holotipo e Isotipo

\$ Zinnia acerosa (DC.) A. Gray var. guanajuatensis Calderón \& Rzedowski, Acta Bot. Mex. 36: 78. 1996. GUANAJUATO: municipio de San Luis de la Paz: cerca de Pregón, 22.VIII.1988, J. Rzedowski 47043. Holotipo e Isotipo

† Zinnia zamudiana Calderón \& Rzedowski, Acta Bot. Mex. 36: 79. 1996. QUERÉTARO: municipio de Cadereyta: $4 \mathrm{~km}$ al ENE de San Javier de las Tuzas, sobre el camino a Sombrerete, 18.VIII.1996, J. Rzedowski 53130. Holotipo e Isotipo

Convolvulaceae

Ipomoea rzedowskii Carranza, Zamudio \& Murgía, Acta Bot. Mex. 45: 32.1998. HIDALGO: municipio de Zimapán: Yethay, 19.VIII.1996, S. Zamudio y E. Pérez 9970. Holotipo e Isotipo

Ipomoea tacambarensis E. Carranza, Sida 20: 1351. 2003. MICHOACÁN: municipio de Tacámbaro: $\pm 1 \mathrm{~km}$ al N de Paso de Morelos, 3.IX.2002, E. Carranza y $V$. Steinmann 6392. Holotipo e Isotipo

Crassulaceae

\$ Echeveria calderoniae Pérez-Calix, Acta Bot. Mex. 38: 9. 1997. GUANAJUATO: municipio de Ocampo: 4-5 km al S de Santa Bárbara, 28.VII.1995, E. Pérez y E. Carranza 3164. Holotipo e Isotipo

Echeveria cante Glass \& Mendoza, Cact. Succ. J. (U.S.) 69: 241. 1997. ZACATECAS: between Sombrerete and Fresnillo, ca. $40 \mathrm{~km} \mathrm{NW}$ of Fresnillo, about $1 \mathrm{~km}$ W of highway, in the Sierra de Chapultepec, 19.VI.1994, C. Glass 8073. Holotipo

\$ Pachyphytum garciae Pérez-Calix \& Glass, Acta Bot. Mex. 48: 4. 1999. QUERÉTARO: municipio de Peñamiller: El Zapote, $\pm 4 \mathrm{~km}$ al NW de Río Blanco, 4.II.1997, E. Pérez C. y S. Zamudio 3574. Holotipo e Isotipo

Pachyphytum machucae I. García, C. Glass y M. Cházaro, Acta Bot. Mex. 47: 10. 1999. MICHOACÁN: municipio de Pajacuarán: $2 \mathrm{~km}$ al E de Pajacuarán, Barranca del Agua, cerca de El Cometa, 7.I.1997, I. García Ruiz 4497. Holotipo 
Pachyphytum rzedowskii I. García, E. Pérez-Calix \& Meyrán, An. Inst. Biol., Ser. Bot. 73: 148. 2002. MICHOACÁN: municipio de Tuxpan: cerro Piedra Redonda, al W de Las Caleras, unos $12 \mathrm{~km}$ al W de Tuxpan, 19.XI.1989, R. Torres y $M$. P. Ramirez 13705. Holotipo

+ Sedum clausenii Pérez-Calix, Acta Bot. Mex. 43: 1. 1998. GUANAJUATO: municipio de Victoria: $3.5 \mathrm{~km}$ al $\mathrm{N}$ de Joya Fría, camino a Puerto de Palmas, 25.IV.1997, E. Pérez y E. Carranza 3615. Holotipo e Isotipo

Sedum hintoniorum B. L. Turner, Phytologia 78: 405. 1995. NUEVO LEÓN: municipio de Zaragoza: Cerro El Viejo, 1.X.1993, Hinton et al. 23797. Isotipo

† Sedum mocinianum Pérez-Calix, Acta Bot. Mex. 45: 50. 1998. GUANAJUATO: municipio de Acámbaro: San Luis de los Agustinos, 21.I.1998, E. Pérez, C. Glass y M. Mendoza 3797. Holotipo

Sedum neovolcanicum Pérez-Calix \& I. García, Acta Bot. Mex. 58: 58. 2002. MICHOACÁN: municipio de Tancítaro: Cerro Tancítaro, $27 \mathrm{~km}$ al W de Uruapan en línea recta, Barranca del Agua, $5.7 \mathrm{~km}$ al NE de Tancítaro, 14.XI.1998, I. García R. y G. Hernández 5542. Holotipo e Isotipo

Sedum papillicaulum Nesom, Phytologia 79: 265. 1995. NUEVO LEÓN: municipio de Zaragoza: Sierra de Peña Nevada, Picacho San Onofre (en el ejemplar se cita: Picacho S. Onofre), 18.VI.1979, Hinton et al. 17551. Isotipo

Thompsonella xochipalensis Gual, Peralta \& Pérez-Calix, Acta Bot. Mex. 40: 38. 1997. GUERRERO: municipio de Eduardo Neri: $2 \mathrm{~km}$ al SE de Xochipala, barranca Xoxocoapa, 29.IX.1990, S. Peralta y M. Gual 49. Holotipo

Crossosomataceae

\$ Velascoa recondita Calderón \& Rzedowski, Acta Bot. Mex. 39: 54. 1997. QUERÉTARO: municipio de Landa: 6-7 km al NE de Lagunita de San Diego, 28.VIII.1991, H. Rubio 2578. Holotipo e Isotipo

\section{Cruciferae}

Ornithocarpa torulosa Rollins, Contr. Gray Herb. 198: 6. 1969. DURANGO: 2 miles W of Coyotes along Mexican Highway 40, 5 miles E of El Salto, 25.VII.1967, D. E. Breedlove 15757. Isotipo 
Ocampo y Medellín: Catálogo de Ejemplares Tipo del Herbario IEB

Cucurbitaceae

\$ Cyclanthera jeffreyi Lira \& Rodríguez-Arévalo, Acta Bot. Mex. 48: 12. 1999.

QUERÉTARO: municipio de Pinal de Amoles: 1-2 km al N de Arquitos, 19.IX.1990,

E. Carranza 2759. Holotipo e Isotipo

Parasicyos dieterleae Lira \& Torres, Acta Bot. Mex. 16: 98. 1991. OAXACA: distrito de Teposcolula: municipio de Teposcolula: cerro El Peñasco al S de Teposcolula, 9.IX.1988, R. Torres y M. L. Torres 12318. Isotipo

Sechiopsis tetraptera Dieterle, Contr. Univ. Mich. Herb. 14: 69. 1980. JALISCO: along highway 80, ca. $19 \mathrm{~km} \mathrm{SW}$ of Melaque (en el ejemplar se cita: along the main highway from Guadalajara to Autlán and Barra de Navidad, about $19 \mathrm{~km}$ from Melaque, along road to Autlán), 8-9.XI.1971, Dieterle 4121 (en el ejemplar se cita: J. V. A. Dieterle, W. J. Duffield y W. L. Graham 4124). Isotipo

\section{Cyperaceae}

Carex complanata Torr. \& Hook. ssp. tropicalis Reznicek \& S. González, Contr. Univ. Mich. Herb. 23: 344. 2001. CHIAPAS: municipio de Larraínzar: along road between San Andrés Larraínzar and Puerto Cato, 12.VII.1997, S. González, A. A. Reznicek, M. González y M. Pinedo 10515. Isotipo

Carex congestiflora Reznicek \& S. González, Contr. Univ. Mich. Herb. 22: 125. 1999. CHIAPAS: municipio de Tenejapa: ca. $1 \mathrm{~km} \mathrm{~S}$ of Tenejapa (Km 26.7) along road to San Cristóbal de las Casas, 10.VII.1997, S. González, A. A. Reznicek, M. González y M. Pinedo 10472. Holotipo e Isotipo

\$ Carex evadens S. González \& Reznicek, Acta Bot. Mex. 40: 19. 1997. QUERÉTARO: municipio de Jalpan: 3-4 km al S de La Parada, El Pilón, 24.IV.1991, B. Servín 994. Holotipo

\$ Carex pinophila Reznicek \& S. González, Contr. Univ. Mich. Herb. 21: 294. 1997. QUERÉTARO: municipio de Pinal de Amoles: ca. $2 \mathrm{~km}$ SW of Pinal de Amoles on road (Hwy 120) to San Juan del Río, 15.VII.1996, S. González, A. A. Reznicek y M. Pinedo 10293. Holotipo

\$ Carex queretarensis Reznicek \& S. González, Contr. Univ. Mich. Herb. 21: 291. 1997. QUERÉTARO: municipio de Landa: ca. 7.5 (air) km SW of El Lobo (km 226) on road (Hwy 120) to Jalpan, between El Lobo and Landa, 14.VII.1996, S. González, A. A. Reznicek y M. Pinedo 10284. Holotipo 
† Carex rzedowskii Reznicek \& S. González, Contr. Univ. Mich. Herb. 20: 218. 1995. MICHOACÁN: municipio de Morelia: alrededores de Loma Caliente, próxima a Umécuaro, 27.XI.1991, J. Rzedowski 51351. Holotipo e Isotipo

Carex tenejapensis Reznicek \& S. González, Contr. Univ. Mich. Herb. 22: 121. 1999. CHIAPAS: municipio de Tenejapa: ca. $1 \mathrm{~km} \mathrm{~S}$ of Tenejapa (Km 26.7) along road to San Cristóbal de las Casas, 10.VII.1997, S. González, A. A. Reznicek, M. González y M. Pinedo 10467. Holotipo e Isotipo

Eleocharis rzedowskii S. González, Phytologia 57: 381. 1985. NUEVO LEÓN: municipio de Galeana: Laguna de Labradores, 29.VIII.1984, S. González y J. Tena 2946. Isotipo

Eleocharis svensoniana S. González, Phytologia 57: 383. 1985. DURANGO: municipio de El Mezquital: $31 \mathrm{~km}$ de La Guajolota, por el camino a Los Charcos, 8.X.1983, S. González, M. González y S. Acevedo 2696. Isotipo

Dioscoreaceae

Dioscorea chamela McVaugh, Fl. Novo-Galiciana 15: 364. 1989. JALISCO: steep hillsides ca. 12-13 km SW of Pihuamo, 19.XI.1970, R. McVaugh, W. L. Graham y $W$. D. Stevens 24447. Isotipo

Dioscorea oreodoxa B. G. Schubert, Fl. Novo-Galiciana 15: 378. 1989. COLIMA: mountain summits near pass ca. 11 miles SSW of Colima on Manzanillo road, 10.VIII.1957, R. McVaugh, J. T. Mickel y M. R. McVaugh 16041. Isotipo

\section{Ebenaceae}

† Diospyros xolocotzii Madrigal \& Rzedowski, Acta Bot. Mex. 1: 3. 1988. MICHOACÁN: municipio de Morelia: La Mintzita, $10 \mathrm{~km}$ al SW de la ciudad de Morelia, 15.IV.1987, X. Madrigal 4245. Holotipo

Ericaceae

Agarista villarrealana González V., Acta Bot. Mex. 5: 13. 1989. JALISCO: municipio de Talpa de Allende: El Caracol, al W de la mina Zimapán, 24.V.1987, L. M. González V. 3092. Isotipo 
Arbutus madrensis S. González, Acta Bot. Mex. 17: 8. 1992. DURANGO: municipio de Durango: Pico La Grulla, Predio Las Bayas (UJED), 15.VII.1990, S. González, A. García y S. Acevedo 4577. Isotipo

Euphorbiaceae

Argythamnia guatemalensis Muell. Arg. var. barrancana McVaugh, Contr. Univ. Mich. Herb. 20: 190. 1995. ZACATECAS: ca. 20-21 km S of Jalpa, 9.IX.1967, $R$. McVaugh y $R$. B. McVaugh 23801. Isotipo

Chamaesyce salsuginosa McVaugh, Contr. Univ. Mich. Herb. 20: 196. 1995. JALISCO: municipio de Villa Corona: wet pasture near Villa Corona, 18.IX.1969, J. V. A. Dieterle 3492. Isotipo

Croton disjuntus V. W. Steinmann, Aliso 19: 185. 2001. CHIHUAHUA: approximately 24 road miles W of Durango state line on highway to Hidalgo del Parral and E of Río de Balleza Canyon, 15.VII.1996, Wilson 96-213 (en el ejemplar se cita: M. F. Wilson y M. A. Wells 96-213). Isotipo

Croton ramillatus Croizat var. magniglandulifer V. W. Steinmann, Aliso 19: 184. 2001. MORELOS: municipio de Tlaquiltenango: Sierra de Huautla, ca. 1 km (by air) S of Huaxtla, 23.IX.1995, Steinmann 798 (en el ejemplar se cita: $V$. W. Steinmann, V. E. Ashworth, R. Cerros, J. T. Columbus y L. M. Zahn 798). Isotipo

Croton varelae V. W. Steinmann, Aliso 19: 182. 2001. NAYARIT: municipio de Nayar: ca. $1 \mathrm{~km} \mathrm{~N}$ of Mesa de Nayar along the trail to El Congrejo, just before the ascent into the large barranca, 14.X.1996, Steinmann 1063 (en el ejemplar se cita: V. W. Steinmann, L. Varela y C. Ramírez 1063). Isotipo

Euphorbia arteagae Buck \& Huft, J. Arnold Arbor. 58: 343. 1977. MICHOACÁN: ca. $75 \mathrm{~km} \mathrm{~N}$ of Arteaga, between Río Tepalcatepec and Arteaga, along the highway $\mathrm{S}$ from Cuatro Caminos (3 km S of Nueva Italia and $30 \mathrm{~km} \mathrm{E}$ of Apatzingán), 24.II.1965, R. McVaugh, M. F. Baad, W. R. Anderson y C. W. Laskowski 22525. Isotipo

Euphorbia galiciana McVaugh (= Euphorbia graminea Jacq. var. novogaliciana McVaugh), Brittonia 13: 173. 1961. JALISCO: Sierra de Manantlán, between Aserradero San Miguel Uno and Durazno (en el ejemplar se cita: Sierra de Manantlán (15-20 miles SE of Autlán), on the bajada S and W of the divide between aserradero San Miguel Uno and Durazno), 7.XI.1952, McVaugh 13985 (en el ejemplar se cita: R. McVaugh y J. Sooby, Jr. 13985). Isotipo 
Euphorbia gradyi V. W. Steinmann \& Ramírez-Roa, Haseltonia 6: 102. 1999. OAXACA: municipio de Teotitlán: along MEX 131, 7 km (by road) S of Tecomavaca, 22.IX.1995, Steinmann 784 (en el ejemplar se cita: V. W. Steinmann, V. E. Ashworth, R. Cerros, J. T. Columbus y L. M. Zahn 784). Isotipo

Euphorbia graminea Jacq. f. foliosa McVaugh, Contr. Univ. Mich. Herb. 19: 225. 1993. NAYARIT: precipitous mountainsides 9.5 miles W of Tepic, 11.IX.1960, $R$. McVaugh, C. Feddema y R. W. Pippen 18943. Isotipo

Euphorbia leucophylla Benth. ssp. comcaacorum V. W. Steinmann \& Felger, Aliso 16: 43. 1997. SONORA: municipio de Hermosillo: Bahía Kino, ca. 3 km N of the point where the highway starts to run parallel to the coast, 1.VI.1996, Steinmann 911 (en el ejemplar se cita: V. W. Steinmann, A. L. Reina y T. R. Van Devender 911). Isotipo

Euphorbia nayarensis V. W. Steinmann, Aliso 19: 181. 2001. NAYARIT: municipio de Nayar: along the road from Ruiz to Mesa del Nayar, $2.5 \mathrm{~km}$ (by road) NE of El Maguey, 13.X.1996, Steinmann 1050 (en el ejemplar se cita: $V$. W. Steinmann y L. Varela 1050). Isotipo

Euphorbia perlignea McVaugh, Brittonia 13: 185. 1961. COLIMA: low mountain summits 7 miles N of Santiago, on the road to Durazno, 10.XII.1959, R. McVaugh y W. N. Koelz 1670. Isotipo

\$ Stillingia pietatis McVaugh, Contr. Univ. Mich. Herb. 20: 210. 1995. MICHOACÁN: municipio de La Piedad: Cerro Grande de La Piedad, 30.VIII.1994, S. Zamudio, R. González-Tamayo y E. Pérez 9352. Holotipo e Isotipo

Tragia pacifica McVaugh, Brittonia 13: 203. 1961. COLIMA: near km. 293, about 15 miles SE of Manzanillo, 9.XII.1959, R. McVaugh y W. N. Koelz 1624. Isotipo

Gentianaceae

+ Geniostemon atarjanum B. L. Turner, Phytologia 76: 9. 1994. GUANAJUATO: municipio de Santa Catarina: El Charco, $12 \mathrm{~km}$ SE de Atarjea, 10.XI.1988, E. Ventura y E. López 6361. Isotipo

Geniostemon gypsophilum B. L. Turner, Phytologia 76: 11. 1994. NUEVO LEÓN: municipio de Aramberri: San Francisco (en el ejemplar se cita: near San Francisco), 26.VIII.1992, Hinton et al. 22354. Isotipo 
\$ Geniostemon rotundifolius Rzedowski \& Calderón, Acta Bot. Mex. 32: 2. 1995. QUERÉTARO: municipio de Jalpan: 3-4 km al S de La Parada, El Pilón, 24.IV.1991, B. Servín 993. Holotipo e Isotipo

Gentiana durangensis Villarreal, Acta Bot. Mex. 34: 49. 1996. DURANGO: municipio de Canelas: $28.5 \mathrm{~km}$ de Canelas, por el camino a Durango, 6.X.1985, S. González 3464. Isotipo

Gentiana hintoniorum B. L. Turner, Phytologia 80: 118. 1996. NUEVO LEÓN: municipio de Aramberri: Cerro El Viejo, 19.XI.1993, Hinton et al. 23961. Isotipo

\$ Gentiana ovatiloba Kusn. ssp. michoacana Villarreal, Acta Bot. Mex. 41: 27. 1997. MICHOACÁN: municipio de Zinapécuaro: lado SE del Cerro San Andrés, 9.XII.1988, M. J. Jasso 666. Isotipo

Gramineae

Festuca roblensis González-Ledesma, Novon 8: 147. 1998. ZACATECAS: municipio de Jerez: rancho El Roble, $19 \mathrm{~km}$ de El Derramadero por terracería a Monte de los García, 7.IX.1993, M. González-Ledesma y J. García P. 530. Isotipo

Muhlenbergia durangensis Herrera, Phytologia 63: 457. 1987. DURANGO: municipio de El Mezquital: $32 \mathrm{~km}$ de Los Charcos, por el camino a La Guajolota, 15.III.1985, M. González et al. 1659. Isotipo

Muhlenbergia jaime-hintonii P. M. Peterson \& J. Valdés Reyna, Sida 18: 686. 1999. NUEVO LEÓN: municipio de Zaragoza: La Joya, Cuesta Blanca, aprox. $15 \mathrm{~km}$ de Aramberri rumbo a Zaragoza, 31.X.1998, J. Valdés Reyna y M. A. Carranza VR2560. Isotipo

Triniochloa gracilis Gómez-Sánchez \& González-Ledesma, Novon 8: 144. 1998. GUERRERO: municipio de General Heliodoro Castillo: $62 \mathrm{~km}$ de Filo de Caballo por terracería a Puerto del Gallo (7 km adelante de El Jilguero), 4.XII.1993, M. González-Ledesma y C. Catalán 553. Isotipo

Iridaceae

Sisyrinchium abietum McVaugh, Fl. Novo-Galiciana 15: 314. 1989. JALISCO: Sierra de Manantlán, seaward-facing slopes (above Durazno), about "2 mi" from Aserradero San Miguel Uno (en el ejemplar se cita: Sierra de Manantlán (15-20 miles SE of Autlán), about 2 miles from Aserradero San Miguel Uno, W and S of the divide toward Manzanillo), 4-5.XI.1952, McVaugh 13868 (en el ejemplar se cita: R. McVaugh y J. Sooby, Jr. 13868). Isotipo 
+ Sisyrinchium arguellesiae Ceja, Espejo \& López-Ferrari, Acta Bot. Mex. 44: 88. 1998. QUERÉTARO: municipio de Pinal de Amoles: 1 km al SW de Pinal de Amoles, sobre la carretera a Vizarrón, 15.III.1987, J. Rzedowski 42685. Isotipo

Sisyrinchium cholewae Espejo, López-Ferrari \& Ceja, Acta Bot. Mex. 49: 20. 1999. DURANGO: municipio de Pueblo Nuevo: km 152 carretera El Salto - Mazatlán, 1 km después de Buenos Aires, 29.VII.1991, A. R. López-Ferrari y A. Espejo 1514. Isotipo

Sisyrinchium conzattii Calderón \& Rzedowski, Phytologia 58: 372. 1985. ESTADO DE MÉXICO: municipio de Tlalmanalco: alrededores del Llano Grande, arriba de San Rafael, 19.VIII.1984, J. Rzedowski 38463. Isotipo

Sisyrinchium hintoniorum Nesom, Phytologia 76: 468. 1994. NUEVO LEÓN: municipio de Zaragoza: Cerro El Viejo, 10.V.1992 (en el ejemplar se cita: 6.X.1992), Hinton et al. 22440. Isotipo

Sisyrinchium microbracteatum Nesom, Phytologia 76: 470. 1994. NUEVO LEÓN: municipio de Aramberri: Aramberri to El Salitre, 26.X.1993, Hinton et al. 23737. Isotipo

Sisyrinchium planicola Ceja \& Cholewa, Acta Bot. Mex. 55: 22. 2001. OAXACA: distrito de Ixtlán de Juárez: municipio de San Juan Atepec: Llano de las Flores, sobre la carretera Tuxtepec - Oaxaca (en el ejemplar se cita: Llano de las Flores, km 134 de la carretera Tuxtepec - Oaxaca), 7.VIII.1998, A. Espejo, A. R. LópezFerrari y J. Ceja 6000. Isotipo

Tigridia estelae López-Ferrari \& Espejo, Novon 4: 386. 1994. DURANGO: municipio de Pueblo Nuevo: El Madroño, carretera El Salto - Mazatlán (en el ejemplar se cita: municipio de El Salto), 29.VII.1991, A. R. López-Ferrari, A. Espejo y M. Flores 1520. Isotipo

Tigridia mariaetrinitatis Espejo \& López-Ferrari, Acta Bot. Mex. 55: 24. 2001. OAXACA: distrito de Tlaxiaco: municipio de Chalcatongo de Hidalgo: alrededores de Rancho Progreso, 26.VI.2000, A. Espejo, A. R. López-Ferrari, J. Ceja y A. Mendoza R. 6247. Isotipo

Tigridia martinezii Calderón, Bol. Soc. Bot. Méx. 47: 3. 1987. HIDALGO: municipio de El Chico: alrededores del Cerro de las Ventanas, 29.VII.1984, J. Rzedowski 38428. Isotipo 
Tigridia potosina López-Ferrari \& Espejo, Acta Bot. Mex. 61: 36. 2002. SAN LUIS POTOSÍ: municipio de Zaragoza: alrededores de Álvarez, 17.VII.1994, A. Espejo, A. R. López-Ferrari y J. Ceja 5134. Isotipo

Labiatae

Hedeoma quinquenervata Bartlett $(=H$. costatum A. Gray var. costatum), Proc. Amer. Acad. 44: 634. 1909. NUEVO LEÓN: Sierra Madre above Monterrey, 5.IV.1906, C. G. Pringle 10241. Isotipo

Satureja hintoniorum B. L. Turner, Phytologia 75: 411. 1993. NUEVO LEÓN: municipio de Aramberri: along road from La Escondida to San Francisco, 23.VII.1993, Hinton et al. 23059. Isotipo

Scutellaria aramberrana B. L. Turner, Phytologia 76: 352. 1994. NUEVO LEÓN: municipio de Aramberri: Cerro Grande, 10.VIII.1986 (en el ejemplar se cita: 10.VII.1986), Hinton et al. 18999. Isotipo

Stachys herrerana Rzedowski \& Calderón, Acta Bot. Mex. 3: 1. 1988. HIDALGO: municipio de Epazoyucan: $1 \mathrm{~km}$ al S de El Guajolote, 25.VIII.1984, J. Rzedowski 38464. Isotipo

Stachys langmaniae Rzedowski \& Calderón, Acta Bot. Mex. 3: 2. 1988. NUEVO LEÓN: municipio de Zaragoza: Encantada, 17.VI.1979 (en el ejemplar se cita: 18.VI.1979), Hinton et al. 17545. Isotipo

\$ Stachys turneri Rzedowski \& Calderón, Acta Bot. Mex. 32: 4. 1995. GUANAJUATO: municipio de Victoria: Milpilla del Pito, $8 \mathrm{~km}$ al $\mathrm{N}$ de La Joya, 20.XI.1989, E. Ventura y E. López 7675. Holotipo e Isotipo

Stachys vulnerabilis Rzedowski \& Calderón, Acta Bot. Mex. 3: 3. 1988. NUEVO LEÓN: municipio de Galeana: Cerro El Potosí, cerca de la cima, 7.VII.1987, S. González 4029. Isotipo

Leguminosae

Bauhinia ramirezii Reynoso, Acta Bot. Mex. 20: 53. 1992. JALISCO: municipio de San Sebastián del Oeste: brecha Las Palmas - San Sebastián, en los límites con el municipio de Puerto Vallarta, 27.VIII.1990 (en el ejemplar se cita: 27.VII.1990), $J$. Reynoso, R. Ramírez D. у C. Ramírez S. 481. Isotipo 
† Dalbergia palo-escrito Rzedowski \& Guridi-Gómez, Acta Bot. Mex. 4: 2. 1988. QUERÉTARO: municipio de Jalpan: La Parada, 23.VI.1988, S. Zamudio 5616. Isotipo

† Inga huastecana M. Sousa, Acta Bot. Mex. 31: 51. 1995. QUERÉTARO: municipio de Landa: $1 \mathrm{~km}$ al NW de San Onofre, 3.VIII.1991, H. Rubio 2524. Isotipo

Lupinus hintoniorum B. L. Turner, Phytologia 76: 295. 1994. NUEVO LEÓN: municipio de Zaragoza: Cerro El Viejo, 17.VI.1993, Hinton et al. 22936. Isotipo

Lupinus jaimehintonianus B. L. Turner (publicado como Lupinus jaimehintoniana), Phytologia 79: 102. 1995. OAXACA: municipio de Miahuatlán: Cerro Quiexobra (en el ejemplar se cita: Quiexobra), 15.X.1995, Hinton et al. 26160. Isotipo

Rhynchosia elisae O. Téllez, Contr. Univ. Mich. Herb. 20: 235. 1995. NAYARIT: municipio de Tepic: $\mathrm{km} 5.4$ de la terracería a El Cuarenteño, que empieza a $500 \mathrm{~m}$ al W del Izote, carretera a Jalcocotán, 29.I.1990, O. Téllez 12583. Isotipo

Sophora juanhintoniana B. L. Turner, Phytologia 76: 385. 1994. NUEVO LÉON: municipio de Aramberri: La Soledad (en el ejemplar se cita: La Soledad, Nr.), 26.III.1994, Hinton et al. 34053. Isotipo

Tephrosia microcarpa O. Téllez, Contr. Univ. Mich. Herb. 20: 231. 1995. NAYARIT: a $10 \mathrm{~km}$ al NE de Francisco I. Madero, camino a Pochotitán, 4.II.1989, O. Téllez y G. Flores F. 11780. Isotipo

Tephrosia sousae O. Téllez, Contr. Univ. Mich. Herb. 20: 233. 1995. NAYARIT: municipio de Ruíz: a 7 km al E de Venados camino a Real de Zopilote, 7.II.1987, O. Téllez y P. Magaña 8272. Isotipo

Lentibulariaceae

+ Pinguicula calderoniae Zamudio, Bol. Soc. Bot. Méx. 68: 85. 2001. QUERÉTARO: municipio de Landa: Llano Chiquito, 14.VI.2000 (en el ejemplar se cita: 13.VI.2000), S. Zamudio, G. Ocampo y A. Reznicek 11421. Holotipo e Isotipo

Pinguicula conzattii Zamudio \& van Marm, Acta Bot. Mex. 62: 16. 2003. OAXACA: municipio de Santo Tomás Ocotepec: alrededores de la Cueva de la Hoya, $\pm 10 \mathrm{~km}$ NE de Santiago Nuyoo, 20.XI.1998, S. Zamudio y G. Ocampo 10933. Holotipo e Isotipo 
Ocampo y Medellín: Catálogo de Ejemplares Tipo del Herbario IEB

Pinguicula crassifolia Zamudio, Acta Bot. Mex. 3. 21. 1988. HIDALGO: municipio de El Chico: Las Ventanas, 11.IV.1976, M. Medina C. 316 (en el ejemplar se cita: 1316). Isotipo

Pinguicula elizabethiae Zamudio, Acta Bot. Mex. 47: 16. 1999. HIDALGO: municipio de Zimapán: arroyo Tolimán, 29.IX.1996, S. Zamudio y E. Pérez C. 10029. Holotipo e Isotipo

Pinguicula emarginata Zamudio \& Rzedowski, Phytologia 60: 258. 1986. VERACRUZ: municipio de Atzalan: Tatzayanala, 10.I.1970, F. Ventura A. 347. Isotipo

Pinguicula gracilis Zamudio, Acta Bot. Mex. 3: 25. 1988. NUEVO LEÓN: municipio de Monterrey: ladera N del Cerro de las Mitras, 19.II.1987, S. Zamudio y S. González 5184. Holotipo

Pinguicula hemiepiphytica Zamudio \& Rzedowski, Acta Bot. Mex. 14: 24. 1991: OAXACA: $58 \mathrm{~km}$ al N de Ixtlán, sobre la carretera a Valle Nacional, 12.X.1987 (en el ejemplar se cita: 12.IX.1987), S. Zamudio 5630. Holotipo e Isotipo

Pinguicula immaculata Zamudio \& Lux, Acta Bot. Mex. 20: 40. 1992. NUEVO LEÓN: municipio de Galeana: km 10 de la brecha Rayones - Galeana, 26.II.1991, S. Zamudio, E. Pérez-Calix y A. García-Arévalo 6225. Holotipo e Isotipo

Pinguicula medusina Zamudio \& Studnicka, Acta Bot. Mex. 53: 68. 2000. OAXACA: municipio de Juxtlahuaca: Laguna Encantada, $\pm 3 \mathrm{~km}$ al $\mathrm{N}$ de Santiago Juxtlahuaca, 27.V.1999, S. Zamudio y G. Ocampo 11050. Holotipo e Isotipo

+ Pinguicula moctezumae Zamudio \& R. Z. Ortega, Acta Bot. Mex. 28: 58. 1994. QUERÉTARO: municipio de Cadereyta: $\pm 6 \mathrm{~km}$ al $\mathrm{S}$ de Las Moras (Casa de Máquinas) por el río Moctezuma, 3.III.1994, E. Pérez C. 2904. Holotipo e Isotipo

Pinguicula moranensis H.B.K. var. neovolcanica Zamudio, Acta Bot. Mex. 49: 27. 1999. HIDALGO: municipio de Real del Monte: Barrio Escobar, cerca de la antigua mina de Morán, 17.VIII.1993, S. Zamudio 9144. Holotipo e Isotipo

Pinguicula takakii Zamudio \& Rzedowski, Phytologia 60: 260. 1986. SAN LUIS POTOSÍ: municipio de Villa de Juárez: Minas de Guascamá, $2 \mathrm{~km}$ al SE de Buenavista, 18.XII.1980, S. Zamudio 3789. Isotipo 
Pinguicula utricularioides Zamudio \& Rzedowski, Acta Bot. Mex. 14: 28. 1991. OAXACA: Cerro Azul, al N de Niltepec, 7.III.1956, T. McDougall s. n. Isotipo

Liliaceae

+ Beaucarnea compacta L. Hernández \& Zamudio, Brittonia 55: 226. 2003. GUANAJUATO: municipio de Atarjea: $6.5 \mathrm{~km}$ NE of El Guamúchil, by dirt road to Atarjea (en el ejemplar se cita: $6.5 \mathrm{~km}$ al SE del Guamúchil, por la brecha a Atarjea), 12.IX.1997, S. Zamudio, E. Pérez C. y L. Hernández 10465. Holotipo e Isotipo

+ Calibanus glassianus L. Hernández \& Zamudio, Brittonia 55: 228. 2003. GUANAJUATO: municipio de Xichú: $10 \mathrm{~km}$ NE of Xichú by the road to Atarjea (en el ejemplar la descripción de la localidad se encuentra en español), 12.IX.1997, E. Pérez C., S. Zamudio y L. Hernández 3719. Holotipo e Isotipo

Dandya balsensis López-Ferrari \& Espejo, Acta Bot. Mex. 18: 11. 1992. MORELOS: municipio de Tlalquiltenango: camino entre Valle de Vázquez y Chimalacatlán, 25.VI.1989, A. Flores C. y D. Martínez A. 1075. Isotipo

Dasylirion gentryi Bogler, Brittonia 50: 71. 1998. SONORA: municipio de Yécora: rocky hillside along highway $16,0.5 \mathrm{mi} \mathrm{W}$ of the Chihuahua - Sonora border, 5.VIII.1997, D. J. Bogler y J. Bogler 1145. Isotipo

Echeandia imbricata Cruden, Contr. Univ. Mich. Herb. 16: 133. 1987. JALISCO: precipitous south-facing mountainsides 4 miles NNE of Talpa de Allende, 1213.X.1960, R. McVaugh, C. Feddema y R. W. Pippen 20109. Isotipo

Echeandia magnifica López-Ferrari, Espejo \& Ceja, Novon 12: 77. 2002. GUERRERO: municipio de Chilpancingo: ca. $2 \mathrm{~km}$ después de Xocomanatlán, rumbo a Omiltemi, 4.IX.1999, J. Ceja, A. Espejo, A. R. López-Ferrari y A. Mendoza R. 907. Isotipo

Maianthemum comaltepecense Espejo, López-Ferrari \& Ceja, Acta Bot. Mex. 36: 22. 1996. OAXACA: distrito de Ixtlán de Juárez: municipio de Santiago Comaltepec: Cerro Pelón, ca. 4 km (en línea recta) al NE de Santiago Comaltepec, 10.IX.1995, A. R. López-Ferrari, A. Espejo y J. Ceja 2125. Isotipo

Maianthemum mexicanum A. García-Arévalo, Acta Bot. Mex. 17: 19. 1992. DURANGO: municipio de Durango: Predio Las Bayas de la Universidad Juárez del Estado de Durango, arroyo La Tecolota, 16.VII.1991, A. García y S. Acevedo 1008. Isotipo 
Schoenocaulon lauricola McVaugh ex Frame, Fl. Novo-Galiciana 15: 267. 1989. AGUASCALIENTES: Sierra del Laurel, near the Jalisco - Aguascalientes border, ca. 10 miles SE of Calvillo (3 hours by horse from rancho de Los Adobes), 2628.VIII.1960, R. McVaugh, C. Feddema y R. W. Pippen 18448. Isotipo

† Yucca queretaroensis Piña, Cact. Suc. Mex. 34: 52. 1989. QUERÉTARO: municipio de Pinal de Amoles: $5 \mathrm{~km}$ al N del poblado de Bucareli, sin fecha, I. Piña s. n. Isotipo

\$ Zigadenus neglectus Espejo, López-Ferrari \& Ceja, Acta Bot. Mex. 48: 47. 1999. GUANAJUATO: municipio de Xichú: Puerto del Manzanar, Sierra de Xichú, 9.IX.1991, E. Ventura y E. López 9560. Isotipo

Magnoliaceae

Magnolia pacifica A. Vázquez ssp. tarahumara A. Vázquez, Brittonia 46: 14. 1994. SONORA: municipio de Álamos: arroyo Corohui, N slope of Sierra Saguaribo, San Bernardo, 15.VI.1989, A. Vázquez, M. Rosales y L. Hernández 5000a. Isotipo

Malvaceae

Abutilon macvaughii Fryxell, Madroño 23: 332. 1976. JALISCO: 8 km E of Chamela (en el ejemplar se cita: "Centro de Investigación y Experimentación de la UNAM", 8 km E of Chamela), 8-10.XII.1970, R. McVaugh, W. L. Graham y W. D. Stevens 25118. Isotipo

Hibiscus colimensis Fryxell, U.S. Dep. Agr. Tech. Bull. 1624: 35. 1980. COLIMA: dry hills 15-25 km NW of Santiago, road to Cihuatlán, Jalisco, 16.III.1965, $R$. McVaugh, M. F. Baad, W. R. Anderson y C. W. Laskowski 23030. Isotipo

Kosteletzkya ramosa Fryxell, Phytologia 37: 294. 1977. JALISCO: roadside ditch 1 mile E of Ayo el Chico, 23.VIII.1958, R. McVaugh, H. F. Loveland y $R$. $W$. Pippen 17230. Isotipo

Kosteletzkya reclinata Fryxell, Phytologia 37: 294. 1977. JALISCO: near km 158, road from Zapotlanejo, ca. 7 miles WNW of Tototlán (en el ejemplar se cita: near $\mathrm{km}$ 58, road from Zapotlanejo, ca. 7 miles WNW of Tototlán), 24.VIII.1958, $R$. McVaugh, H. F. Loveland y R. W. Pippen 17259. Isotipo 
Robinsonella macvaughii Fryxell, Gentes Herb. 11: 24. 1973. JALISCO: dry hills between Barra de Navidad and Playa de Cuastecomate, 16.III.1965, R. McVaugh, M. F. Baad, W. R. Anderson y C. W. Laskowski 23038. Isotipo

Melastomataceae

Arthrostemma primaevum Almeda, Brittonia 46: 76. 1994. HIDALGO: municipio de Tenango de Doria: roadbank $5 \mathrm{~km}$ NE of Tenango de Doria, 30.X.1983, D. E. Breedlove y F. Almeda 59534. Isotipo

Menispermaceae

Menispermum mexicanum Rose (= M. canadense L.), Contr. U.S. Nat. Herb. 13: 302. 1911. NUEVO LEÓN: Sierra Madre above Monterrey, 8.VII.1907, C. G. Pringle 10378. Isotipo

Myrtaceae

Eugenia uxpanapensis P. E. Sánchez \& L. M. Ortega, Phytologia 63: 404. 1987. VERACRUZ: municipio de Hidalgotitlán: Río Solosúchil, SW del campamento Hermanos Cedillo, 2.IV.1974, M. Vázquez 293. Isotipo

Nyctaginaceae

Boerhavia traubae Spellenberg, Madroño 46: 208. 1999. SONORA: municipio de Yécora: $1.6 \mathrm{~km}$ E of Yécora on Mex. Hwy. 16 at km marker 281.5, 15.VIII.1998, R. Spellenberg, L. Brouillet y T. K. Todsen 12597. Isotipo

Commicarpus coctoris N. A. Harriman, Sida 18: 679. 1999: OAXACA: municipio de San Agustín Atenango: $14 \mathrm{~km}$ al SW de San Marcos Arteaga, carretera Huajuapan - Juxtlahuaca, 23.XI.1989, S. D. Koch y P. A. Fryxell 89220. Isotipo

Oleaceae

Menodora gypsophila B. L. Turner, Phytologia 79: 8. 1995. NUEVO LEÓN: municipio de Galeana: Santa Rosa (en el ejemplar se cita: along road from Ciénega del Toro to Santa Rosa), 6.X.1995 (en el ejemplar se cita: 3.X.1995), Hinton et al. 25643. Isotipo 
Ocampo y Medellín: Catálogo de Ejemplares Tipo del Herbario IEB

Onagraceae

Megacorax gracielanus S. González E. \& W. L. Wagner, Novon 12: 361. 2002. DURANGO: municipio de Nuevo Ideal: Sierra de Coneto, al N de Dr. Castillo del Valle, por el camino a Ignacio Zaragoza, 5.XI.2001, S. Acevedo, L. López y S. González E. 1352. Isotipo

Orchidaceae

\$ Bletia campanulata Lex., Nov. Veg. Descr. 2: Orchid. opusc. 17. 1825. (Neotipo designado por V. Sosa en Acta Bot. Mex. 18: 78. 1992). MICHOACÁN: municipio de Acuitzio del Canje: $10 \mathrm{~km}$ adelante de Acuitzio del Canje, rumbo a Villa Madero, 25.VII.1989, A. Espejo у A. R. López-Ferrari 3673. Isoneotipo

Dendrobium bracteatum Lex. (= Isochilus bracteatus (Lex.) Salazar \& Soto Arenas), Nov. Veg. Descr. 2: Orchid. opusc. 43. 1825. (Neotipo designado por A. R. López-Ferrari y A. Espejo en Acta Bot. Mex. 51: 65. 2000). MICHOACÁN: municipio de Uruapan: pedregal entre Uruapan y Tingambato, 18.X.1994, M. A. Soto et al. 7633. Isoneotipo

Galeottiella hintoniorum Todzia, Brittonia 46: 332. 1994. NUEVO LEÓN: municipio de Zaragoza: Cerro Viejo (en el ejemplar se cita: Cerro El Viejo), 20.II.1993, Hinton et al. 22636. Isotipo

† Habenaria zamudioana González-Tamayo, Bol. Inst. Bot. (Guadalajara) 3: 71. 1995. MICHOACÁN: municipio de La Piedad: parte alta del Cerro de Cujuarato (Cerro Grande) (en el ejemplar se cita: Cerro Grande de la Piedad), 30.VIII.1994, S. Zamudio, R. González y E. Pérez 9353. Holotipo e Isotipo

Liparis volcanica González-Tamayo \& Zamudio, Acta Bot. Mex. 23: 60. 1993. MICHOACÁN: municipio de Uruapan: $2 \mathrm{~km}$ al N de Caltzontzin, 2.IX.1991, E. Pérez y S. Zamudio 2594. Holotipo e Isotipo

Malaxis rzedowskiana González-Tamayo, Bol. Inst. Bot. (Guadalajara) 8: 175. 2001. ESTADO DE MÉXICO: municipio de Texcoco: 5 km al E de Coatlinchán, 3.VIII.1981, J. Rzedowski 37458. Holotipo

\$ Platanthera calderoniae López-Ferrari \& Espejo, Acta Bot. Mex. 26: 77. 1994. MICHOACÁN: municipio de Morelia: $2.5 \mathrm{~km}$ después de San Miguel del Monte, rumbo a Túmbisca, 5.III.1993, A. Espejo y A. R. López-Ferrari 4882. Isotipo 
Acta Botanica Mexicana 67: 1-41 (2004)

Papaveraceae

Hunnemannia hintoniorum Nesom, Phytologia 73: 335. 1992. NUEVO LEÓN: municipio de Galeana: near Río de San José (en el ejemplar se cita: near San José del Río), 24.III.1992, Hinton et al. 21876. Isotipo

Piperaceae

Peperomia calderoniae Barrios, Cota \& Medina-Cota, Phytologia 62: 54. 1987. HIDALGO: municipio de Epazoyucan: $1 \mathrm{~km}$ al SW de El Guajolote, 17.VI.1984, $M$. Medina-Cota, H. Cota y M. Barrios 2572. Isotipo

Podostemaceae

Marathrum rubrum Novelo \& Philbrick, Novon 3: 456. 1993. JALISCO: municipio de Cabo Corrientes: Puente Los Horcones sobre el río, a $27 \mathrm{~km}$ al S de Puerto Vallarta rumbo a Chamela, 6.III.1991, A. Novelo y T. Philbrick 1003. Isotipo

Oserya longifolia Novelo \& Philbrick, Novon 5: 54. 1995. JALISCO: municipio de La Huerta: río Purificación, $4 \mathrm{~km}$ al W de La Huerta, 26.III.1993, A. Novelo y T. Philbrick 1166. Isotipo

Polemoniaceae

† Polemonium melindae Rzedowski, Calderón \& Villarreal, Acta Bot. Mex. 31: 58. 1995. QUERÉTARO: municipio de Colón: parte alta del Cerro Zamorano, 10.V.1987, J. Rzedowski 43240. Holotipo e Isotipo

Portulacaceae

* Portulaca guanajuatensis G. Ocampo, Acta Bot. Mex. 58: 1. 2002. GUANAJUATO: municipio de Iturbide: km 9 de la carretera San José Iturbide Tierra Blanca, 22.IX.2001, G. Ocampo y A. Medellín 1152. Holotipo e Isotipo

\$ Portulaca matthewsii G. Ocampo, Sida 20: 1358. 2003. QUERÉTARO: municipio de Landa: Puerto del Gato, $3 \mathrm{~km}$ al W de Acatitlán de Zaragoza, 29.VII.1988, E. González 44. Holotipo

† Portulaca rzedowskiana G. Ocampo, Sida 20: 487. 2002. MICHOACÁN: municipio de Morelia: Monte Rubio, 1.VIII.1991, J. M. Escobedo 2035. Holotipo e Isotipo 
Ocampo y Medellín: Catálogo de Ejemplares Tipo del Herbario IEB

Rosaceae

† Rubus macvaughianus Rzedowski \& Calderón, Acta Bot. Mex. 5: 1. 1989. QUERÉTARO: municipio de Colón: parte alta del Cerro Zamorano, 1.IX.1987, J. Rzedowski 44377. Isotipo

Rubiaceae

Galium hintoniorum B. L. Turner, Phytologia 80: 285. 1996. TAMAULIPAS: municipio de Güemes: Los Pedros, 10.XI.1994, Hinton et al. 25127. Isotipo

Hedyotis nigricans (Lam.) Fosberg var. gypsophila B. L. Turner, Phytologia 79: 15. 1995. NUEVO LEÓN: municipio de Galeana: Santa Rita, 11.VI.1981, Hinton et al. 18278. Isotipo

Salicaceae

Populus guzmanantlensis Vázquez \& Cuevas, Acta Bot. Mex. 8: 39. 1989. JALISCO: municipio de Casimiro Castillo: 1-2 km al NE de Casimiro Castillo, 14.X.1988, R. Cuevas y L. Guzmán 3224. Isotipo

+ Salix aeruginosa E. Carranza, Acta Bot. Mex. 32: 33. 1995. MICHOACÁN: municipio de Charo: alrededores del Parque José María Morelos ("km 23"), al E de Morelia, 7.IV.1994, E. Carranza 4762. Holotipo

Saxifragaceae

Pterostemon bravoanus Jiménez Ramírez \& Martínez Gordillo, Acta Bot. Mex. 41: 21. 1997. GUERRERO: municipio de Eduardo Neri: $8 \mathrm{~km}$ al NE de Carrizalillo, camino a Mezcala, 7.X.1996, R. Cruz Durán 975 (en el ejemplar se cita: R. Cruz Durán y Ma. E. García 975). Isotipo

\$ Ribes grandisepalum C. Durán \& S. Avendaño (= Ribes ciliatum Humb. \& Bonpl. ex Roem. \& Schult.), Acta Bot. Mex. 60: 14. 2002. MICHOACÁN: municipio de Zinapécuaro: $1 \mathrm{~km}$ al NW de Laguna Larga, sobre el camino a Yerbabuena, 13.III.1988, J. Rzedowski 46282. Isotipo

Scrophulariaceae

Castilleja galehintoniae Nesom, Phytologia 72: 246. 1992. NUEVO LEÓN: municipio de Galeana: San José de las Joyas, 5.VII.1983, Hinton et al. 18422. Isotipo 
Leucophyllum alejandrae Nesom, Phytologia 74: 293. 1993. NUEVO LEÓN: municipio de Galeana: S of San Antonio de Texas (en el ejemplar se cita: S of San Ignacio de Texas), 19.VIII.1992, Hinton et al. 22321. Isotipo

Leucophyllum hintoniorum Nesom, Phytologia 71: 337. 1991. NUEVO LEÓN. municipio de Aramberri: E of Aramberri (en el ejemplar se cita: W of Aramberri), 14.V.1991, Hinton et al. 20929. Isotipo

Solanaceae

Chamaesaracha rzedowskiana A. T. Hunziker, Contr. Gray Herb. 210: 23. 1980. SAN LUIS POTOSÍ: municipio de Xilitla: Las Crucitas, 1.III.1959, J. Rzedowski 10103. Isotipo

+ Physalis queretaroensis M. Martínez \& L. Hernández, Acta Bot. Mex. 46: 73. 1999. QUERÉTARO: municipio de Landa: $2-5 \mathrm{~km}$ al S del Parador Santa Martha, 16.VII.1990, E. Carranza 2585. Holotipo

Styracaceae

Styrax argenteus Presl var. grandiflorus E. Carranza, Acta Bot. Mex. 36: 15. 1996. OAXACA: municipio de Juxtlahuaca: $3 \mathrm{~km}$ al S de la desviación a San Martín Peras sobre el camino a Coicoyán de las Flores, 27.IX.1993, J. L. Panero, P. Dávila e I. Calzada 3475. Isotipo

+ Styrax argenteus Presl var. parvifolius E. Carranza, Acta Bot. Mex. 23: 36. 1993. QUERÉTARO: municipio de Arroyo Seco: $\pm 2 \mathrm{~km}$ al W de Agua Fría de los Fresnos, 23.IV.1992, E. Carranza 4033. Holotipo e Isotipo

Symplocaceae

\$ Symplocos citrea Lex., Nov. Veg. Descr. 1: 22. 1824. (Neotipo designado por H. Díaz B. y M. Cházaro en Acta Bot. Mex. 23: 43. 1993). MICHOACÁN: municipio de Morelia: Ichaqueo, 23.XI.1992, H. Díaz B. 7260. Neotipo e Isoneotipo

Thymelaeaceae

Dirca mexicana Nesom \& Mayfield, Sida 16: 460. 1995. TAMAULIPAS: municipio de Hidalgo: along road from Sta. Engracia (Tamaulipas) to Dulces Nombres (Nuevo León), Arroyo Oscuro, 2.0 road mi NE of Los Caballos toward Cañada El Mimbre, 
15.0 road mi W of lowermost crossing of arroyo El Mimbre, 3.III.1995, G. Nesom, M. Mayfield y G. Anderson 7863. Isotipo

Ulmaceae

Ulmus ismaelis Todsia \& Panero, Brittonia 50: 343. 1998. OAXACA: distrito de Santiago Juxtlahuaca: municipio de Santos Reyes Tepejillo: al fondo del Cañón La Cruz, $4 \mathrm{~km}$ al N de Santos Reyes Tepejillo sobre la carretera a Santa María Tindú, 16.VI.1996, J. L. Panero y J. I. Calzada 6573. Isotipo

Umbelliferae

\$ Tauschia beruloides Constance \& Affolter, Brittonia 49: 460. 1997. MICHOACÁN: municipio de Zinapécuaro: parte alta del Cerro San Andrés, 29.VIII.1987, S. Zamudio 5574. Holotipo

Valerianaceae

\$ Valeriana emmanuellii Rzedowski \& Calderón, Acta Bot. Mex. 62: 66. 2003. MICHOACÁN: municipio de Morelia: parte alta del Cerro Campanario, $4 \mathrm{~km}$ al SE de Agua Zarca, 27.VII.2002, J. Rzedowski 53966. Holotipo e Isotipo

† Valeriana zamoranensis Rzedowski \& Calderón, Acta Bot. Mex. 62: 69. 2003. QUERÉTARO: municipio de Colón: parte alta del Cerro Zamorano, 27.VIII.1989, J. Rzedowski 48780. Holotipo e Isotipo

Verbenaceae

\$ Priva ibugana Rzedowski \& Calderón, Bol. Inst. Bot. (Guadalajara) 5: 65.1998. GUANAJUATO: municipio de Comonfort: $2 \mathrm{~km}$ al NE de Empalme Escobedo, 27.VI.1987, J. Rzedowski 43445. Holotipo

Violaceae

\$ Viola cochranei Ballard, Phytologia 74: 357. 1993. QUERÉTARO: municipio de San Juan del Río: 2 km al S de Puerto Alegrías, 26.V.1986, R. Fernández 3329. Isotipo

Viola hemsleyana Calderón, Phytologia 52: 101. 1982. ESTADO DE MÉXICO: municipio de Naucalpan: Villa Alpina, 1.VII.1979, J. Rzedowski 36144. Isotipo 


\section{AGRADECIMIENTOS}

Los autores deseamos reconocer la amable ayuda proporcionada por las siguientes personas para conseguir los datos de las colecciones de tipos de algunos herbarios mexicanos: Luis Sánchez (AMO), José Ángel Villarreal (ANSM), Stephen D. Koch (CHAPA), Germán Carnevali y Silvia Hernández (CICY), Rosa Elena Martínez (IBUG), Ma. del Rosario García y Helga Ochotorena (MEXU), Ana Rosa López-Ferrari (UAMIZ) y Francisco Lorea (XAL). De igual manera, agradecemos a Jerzy Rzedowski y a Graciela Calderón de Rzedowski por la revisión del escrito, así como por su apoyo para la realización de este trabajo.

\section{LITERATURA CITADA}

Arreguín, M. L. y R. Valenzuela. 1986. Segundo catálogo de los herbarios institucionales mexicanos. Consejo Nacional de la Flora de México, A.C. México, D.F. 143 pp.

Arreguín, M. L., R. Fernández y A. Rodríguez. 1996. Catálogo de los ejemplares tipo de plantas vasculares del Herbario de la Escuela Nacional de Ciencias Biológicas (ENCB), del Instituto Politécnico Nacional, México. Polibotanica 3: 18-81.

Arreguín, M. L., A. M. Escamilla y R. Fernández. 2003. Adiciones de ejemplares tipo de plantas vasculares depositados en el Herbario de la Escuela Nacional de Ciencias Biológicas del Instituto Politécnico Nacional. Polibotanica 16: 113-123.

Greuter, W., J. McNeill, F. R. Barrie, H. M. Burdet, V. Demoulin, T. S. Filgueiras, D. H. Nicholson, P. C. Silva, J. E. Skog, P. Trehane, N. J. Turland y D. L. Hawksworth. 2000. International code of botanical nomenclature (Saint Louis Code). International Association for Plant Taxonomy. Königstein, Alemania. 474 pp.

Hernández, R. y R. Lira. 1991. Catálogo de ejemplares tipo del Herbario Nacional de México (MEXU). I. Criptógamas y gramíneas. An. Inst. Biol., Ser. Bot. 61(1): 45-63.

Lira, R., S. Arias, S. Gama y U. Guzmán. 1992. Catálogo de ejemplares tipo del Herbario Nacional de México (MEXU). II. Cactaceae y Cucurbitaceae. An. Inst. Biol., Ser. Bot. 63(2): 213-219.

Lira, R., M. R. García y J. L. Villaseñor. 1996. Catálogo de ejemplares tipo del Herbario Nacional de México (MEXU). III. Compositae. An. Inst. Biol., Ser. Bot. 67(2): 385-417.

Llorente, J., P. Koleff, H. Benítez y L. Lara. 1999. Síntesis del estado de las colecciones biológicas mexicanas. Resultado de la encuesta "Inventario y diagnóstico de la actividad taxonómica en México" 1996-1998. Comisión Nacional para el Conocimiento y Uso de la Biodiversidad. México, D.F. 143 pp.

Martínez, R. E. 1993. La colección de ejemplares tipo del Herbario IBUG, Universidad de Guadalajara, México. Bol. Inst. Bot. (Guadalajara) 1(6): 449-459.

Montero, J. C., M. Sousa y M. R. García. 2001. Catálogo de ejemplares tipo del Herbario Nacional de México (MEXU). V. Solanaceae. An. Inst. Biol., Ser. Bot. 72(1): 119-122. 
Ocampo y Medellín: Catálogo de Ejemplares Tipo del Herbario IEB

Olvera, M. 1999. Catálogo de ejemplares tipo del Herbario Nacional de México (MEXU). IV. Lentibulariaceae. An. Inst. Biol., Ser. Bot. 70(2): 159-160.

Recibido en julio de 2003. Aceptado en febrero de 2004. 